COMMUNICATIONS IN

ANALYSIS AND GEOMETRY

Volume 14, Number 2, 283-343, 2006

\title{
Convergence of the Ricci flow toward a soliton
}

\begin{abstract}
Natasa Sesum
We will consider a $\tau$-flow, given by the equation $\frac{d}{d t} g_{i j}=-2 R_{i j}+$ $\frac{1}{\tau} g_{i j}$ on a closed manifold $M$, for all times $t \in[0, \infty)$. We will prove that if the curvature operator and the diameter of $(M, g(t))$ are uniformly bounded along the flow, then we have a sequential convergence of the flow toward the solitons. If we also assume that one of the limit solitons is integrable, then we have a convergence toward a unique soliton, up to a diffeomorphism.
\end{abstract}

\section{Introduction.}

The Ricci flow equation

$$
\frac{d}{d t} g_{i j}=-2 R_{i j}
$$

has been introduced by Hamilton in his seminal paper [6]. We will refer to this equation as to an unnormalized Ricci flow. A normalized Ricci flow is given by the equation

$$
\frac{d}{d t} \tilde{g}_{i j}=-2 R(\tilde{g})_{i j}+\frac{2}{n} r \tilde{g}_{i j}
$$

where $r=\frac{1}{\operatorname{Vol}(M)} \int_{M} R(\tilde{g}) d V_{\tilde{g}}$. This equation is sometimes more convenient to consider, since a volume of a manifold is being fixed along the normalized Ricci flow and a volume collapsing case cannot happen in a limit, if the limit exists.

A natural question that arises in studying the evolution equations, in particular, the Ricci flow equation, is under which conditions a solution will exist for all times, that is under which conditions it will avoid the singularities at finite times. The other question one can ask is if there exists a limit to the solutions when we approach infinity and how we can describe the metrics obtained in the limit. In the case of dimension three with positive Ricci curvature and dimension four with positive curvature operator, we 
know (due to Hamilton) that the solutions of the Ricci flow equation, in both cases exist for all times, converging to Einstein metrics. In general, we cannot expect to get an Einstein metric in the limit. We can expect to get in the limit a solution to the Ricci flow equation which moves under oneparameter subgroup of the symmetry group of the equation. These kinds of solutions are called solitons. Since the Ricci flow equation is a gradient flow of Perelman's functional $\mathcal{W}$, it is natural to expect that a soliton in the limit is unique up to diffeomorphisms.

Our goal in this paper is to prove the following theorem.

Theorem 1.1. Let $\left(g_{i j}\right)_{t}=-2 R_{i j}+\frac{1}{\tau} g_{i j}$ be a Ricci flow on a closed manifold $M$ with uniformly bounded curvature operators and diameters for all $t \in[0, \infty)$. Assume also that some limit soliton is integrable. Then there is an 1-parameter family of diffeomorphisms $\phi(t)$, a unique soliton $h(t)$ and constants $C, \delta, t_{0}$ such that $\left|\phi(t)^{*} g(t)-h(0)\right|_{k, \alpha}<C e^{-\delta t}$, for all $t \in\left[t_{0}, \infty\right)$. Moreover, if $\psi(t)$ is a diffeomorphism such that $h(t)=\psi^{*} h(0)$, then $\left|(\phi \psi)^{*} g(t)-h(t)\right|_{C^{0}}<C e^{-c t}$.

The ideas for the proof of Theorem 1.1 have been inspired by those of Cheeger and Tian in [3].

\section{Outline of the proof of Theorem 1.1}

In order to deal with this problem, we will first construct a gauge on time intervals of an arbitrary length, so that in the chosen gauge, the $\tau$-flow equation becomes strongly parabolic. We will look at the solutions of a strictly parabolic equation. It will turn out that our metrics (in the right gauge) will satisfy a strictly parabolic equation that is almost linear and therefore their behavior is modeled on the behavior of the solutions of the linear equation. There are 3 types of the solutions of our strictly parabolic equation,

- the solutions that have an exponential growth,

- the solutions that have an exponential decay,

- the solutions that change very slowly.

Roughly speaking, the integrability condition means that the solutions of a linearized deformation equation for solitons arise from a curve of metrics satisfying the same soliton equation. To deal with those slowly changing 
solutions, we will use the integrability condition to change the reference soliton metric so that at the end, we deal only with the cases of either a growth or a decay. We will rule out the possibility of the exponential growth, by using the fact that our flow sequentially converges toward solitons and by using the similar arguments established by Simon in [15] and also later used by Cheeger and Tian in [3]. We will be left with the exponential decay which will allow us to continue our gauge up to infinity.

The organization of the paper is as follows. In Section 2, we will give a necessary background and notation. In Section 4, we will prove a sequential convergence of a $\tau$-flow with uniformly bounded curvature operators and diameters toward the solitons. In Section 5, using the sequential convergence of the $\tau$-flow, we will construct a gauge on time intervals of an arbitrary length, so that in the chosen gauge the $\tau$-flow equation becomes strongly parabolic. In Section 6, we will use the integrability assumption to prove that a soliton that we get in the limit is unique up to a diffeomorphism.

\section{Background.}

Perelman's functional $\mathcal{W}$ and its properties will play an important role in the paper. $M$ will always denote a closed manifold. $\mathcal{W}$ has been introduced in $[11]$.

$$
\mathcal{W}(g, f, \tau)=(4 \pi \tau)^{-\frac{n}{2}} \int_{M} e^{-f}\left[\tau\left(|\nabla f|^{2}+R\right)+f-n\right] d V_{g} .
$$

We will consider this functional restricted to $f$ satisfying

$$
\int_{M}(4 \pi \tau)^{-\frac{n}{2}} e^{-f} d V=1
$$

$\mathcal{W}$ is invariant under simultaneous scalings of $\tau$ and $g$ and under a diffeomorphism change, i.e. $\mathcal{W}(g, f, \tau)=\mathcal{W}\left(c \phi^{*} g, \phi^{*} f, c \tau\right)$ for a constant $c>0$ and a diffeomorphism $\phi$. Perelman showed that the Ricci flow can be viewed as a gradient flow of a functional $\mathcal{W}$, which is one of the reasons why this functional plays an important role throughout [11]. Let $\mu(g, \tau)=\inf \mathcal{W}(g, f, \tau)$ over smooth $f$ satisfying (2.1). It has been showed by Perelman that $\mu(g, \tau)$ is achieved by some smooth function $f$ on a closed manifold $M$, that $\mu(g, \tau)$ is negative for small $\tau>0$ and that it tends to zero as $\tau \rightarrow 0$.

We will explain the motivation why we have decided to study this flow instead of a normalized one in which a volume of a manifold has been fixed along the flow. First of all, there is a simple reparametrization that allows us to go from a $\tau$-flow to an unnormalized flow and many smoothing 
regularity properties that have been proved for the unnormalized flow continue to hold for a $\tau$-flow as well. For example, Hamilton's compactness theorem also holds for the $\tau$-flow. This is because Shi's estimates hold for $\tau$-flow as well, and therefore, since we have a uniform curvature bound on the solutions to a $\tau$-flow, we may assume uniform bounds on all covariant derivatives of the curvature, $\left|D^{p} \mathrm{Rm}\right| \leq C(p)$. The reparametrization that we use to go from a $\tau$-flow to an unnormalized flow is as follows. Let $c(s)=1-\frac{s}{\tau}$ and $t(s)=-\tau \ln \left(1-\frac{s}{\tau}\right)$. Let $\tilde{g}(s)=c(s) g(t(s)) . \quad \tilde{g}(s)$ is a solution to an unnormalized Ricci flow. On the other hand, we have that $\mathcal{W}(g(t(s)), f(t(s)), \tau)=\mathcal{W}(\tilde{g}(s), \tilde{f}(s), \tau-s)$. By the monotonicity formula for $\mathcal{W}$, we have that the latter quantity is increasing along an unnormalized Ricci flow and therefore, the former quantity is increasing along the $\tau$ flow as well. The monotonicity formula for a $\tau$-flow gets the simpler form; $\mathcal{W}(g(t), f(t), \tau)$ is increasing along the $\tau$-flow, while $f(t)$ changes by the evolution equation $\frac{d}{d t} f=-\Delta f+|\nabla f|^{2}-R+\frac{n}{2 \tau}$ and $\tau$ is just a constant. The fact that $\tau$ is now a constant will be very useful in taking the limits of the minimizers for $\mathcal{W}$.

One of the most important properties of $\mathcal{W}$ is the monotonicity formula.

Theorem 2.1 (Perelman). $\frac{d}{d t} \mathcal{W}=\int_{M} 2 \tau\left|R_{i j}+\nabla_{i} \nabla_{j} f-\frac{1}{2 \tau} g_{i j}\right|^{2}(4 \pi \tau)^{-\frac{n}{2}}$ $e^{-f} d V \geq 0$ and therefore, $\mathcal{W}$ is increasing along the flow described by the following equations

$$
\begin{aligned}
\frac{d}{d t} g_{i j} & =-2 R_{i j} \\
\frac{d}{d t} f & =-\Delta f+|\nabla f|^{2}-R+\frac{n}{2 \tau} \\
\dot{\tau} & =-1
\end{aligned}
$$

One of the very important applications of the monotonicity formula is non-collapsing theorem for the Ricci flow that has been proved by Perelman in [11].

Definition 2.2. Let $g_{i j}(t)$ be a smooth solution to the Ricci flow $\left(g_{i j}\right)_{t}=$ $-2 R_{i j}(t)$ on $[0, T)$. We say that $g_{i j}(t)$ is locally collapsing at $T$, if there is a sequence of times $t_{k} \rightarrow T$ and a sequence of metric balls $B_{k}=B\left(p_{k}, r_{k}\right)$ at times $t_{k}$, such that $\frac{r_{k} 2}{t_{k}}$ is bounded, $|\operatorname{Rm}|\left(g_{i j}\left(t_{k}\right)\right) \leq r_{k}^{-2}$ in $B_{k}$ and $r_{k}^{-n} \operatorname{Vol}\left(B_{k}\right) \rightarrow 0$. 
Theorem 2.3 (Perelman). If $M$ is closed and $T<\infty$, then $g_{i j}(t)$ is not locally collapsing at $T$.

The corollary of Theorem 2.3 is

Corollary 2.4. Let $g_{i j}(t), t \in[0, T)$ be a solution to the Ricci flow on a closed manifold $M$, where $T<\infty$. Assume that for some sequences $t_{k} \rightarrow T$, $p_{k} \in M$ and some constant $C$ we have $Q_{k}=|\operatorname{Rm}|(x, t) \leq C$, whenever $t<t_{k}$. Then a subsequence of scalings of $g_{i j}\left(t_{k}\right)$ at $p_{k}$ with factors $Q_{k}$ converges to a complete ancient solution to the Ricci flow, which is $\kappa$-noncollapsed on all scales for some $\kappa>0$.

We would like to recall a definition of a soliton that will appear in later sections.

Definition 2.5. A Ricci soliton $g(t)$ is a solution to a Ricci flow equation that moves by 1-parameter group of diffeomorphisms $\phi(t)$, i.e. $g(t)=$ $\phi(t)^{*} g(0)$.

The equation for a metric to move by a diffeomorphism in the direction of a vector field $V$ is $2 \operatorname{Ric}(g)=\mathcal{L}_{V}(g)$, or $R_{i j}=g_{i k} D_{j} V^{k}+g_{j k} D_{i} V^{k}$. If the vector field $V$ is the gradient of a function $f$, we say that the soliton is the gradient Ricci soliton. Moreover, we can consider the solutions to the Ricci flow that move by diffeomorphisms and also shrink or expand by a factor at the same time. The stationary solutions of the unnormalized Ricci flow are the Ricci flat metrics. The Ricci solitons are the generalizations of those, namely they are the stationary solutions to the Ricci flow equations, up to diffeomorphisms.

\section{Sequential convergence of a $\tau$-flow.}

Definition 3.1. $\tau$-flow is given by the equation

$$
\frac{d}{d t} g_{i j}=-2 R_{i j}+\frac{1}{\tau} g_{i j}
$$

for $\tau>0$.

We want to prove the following theorem in this section 
Theorem 3.2. Consider the flow

$$
\frac{d g_{i j}}{d t}=-2 R_{i j}+\frac{1}{\tau} g_{i j}
$$

on a compact manifold $M$, where $\tau>0$ is fixed, $|R m| \leq C$ and $\operatorname{diam}\left(M, g(t) \leq C, \forall t \in[0, \infty)\right.$. Then for every sequence of times $t_{i} \rightarrow \infty$ there exists a subsequence, so that $g\left(t_{i}+t\right) \rightarrow h(t)$ and $h(t)$ is a Ricci soliton.

\subsection{Convergence toward the solutions of the Ricci flow.}

In order to prove Theorem 3.2, we will first show that it is reasonable to expect a convergence toward a smooth manifold, i.e. that a limit manifold will not collapse.

Claim 3.3. Consider the flow as above. For every fixed $\tau>0$, there exists a constant $C$ such that $\operatorname{Vol}_{g(t)}(M) \geq C$ for every $t$, i.e. we have a uniform lower bound on the volumes.

Proof. Assume that the claim is not true, i.e. that there exists a sequence $t_{i}$ s.t. $\operatorname{Vol}_{g\left(t_{i}\right)}(M) \rightarrow 0$ as $i \rightarrow \infty$. Let $\bar{g}(s)=c(s) g(t(s))$ be unnormalized flow, for $s \in[0, \tau)$, where:

$$
\begin{gathered}
t(s)=-\tau \ln \left(1-\frac{s}{\tau}\right) . \\
c(s)=1-\frac{s}{\tau} . \\
R(\bar{g})=\frac{R(g)}{c(s)} .
\end{gathered}
$$

Find $s_{i}$, such that $t\left(s_{i}\right)=t_{i}$. We get that $s_{i}=\tau\left(1-e^{-\frac{t_{i}}{\tau}}\right) . s_{i} \rightarrow \tau$ as $i \rightarrow \infty$. Let

$$
\max _{M \times\left[0, s_{i}\right]}|R m|(\bar{g}(s))=Q_{i},
$$

and assume that the maximum is achieved at $p_{i}$. By the corollary of Perelman's non-collapsing theorem, we have that:

$$
\frac{\operatorname{Vol}_{\bar{g}(t)} B\left(p_{i}, r\right)}{r^{n}} \geq C_{1},
$$


for $r \leq C \sqrt{\frac{\tau}{Q_{i}}}$ and $t \in\left[0, s_{i}\right)$. Choose $r=C \sqrt{\frac{\tau}{Q_{i}}}$ and $t=s_{i}$.

$$
\left(\sqrt{Q_{i}}\right)^{n} \operatorname{Vol}_{\bar{g}\left(s_{i}\right)} B\left(p_{i}, C \sqrt{\frac{\tau}{Q_{i}}}\right) \geq(C \sqrt{\tau})^{n} C_{1}=\tilde{C} .
$$

Since $\operatorname{Vol}_{\bar{g}\left(s_{i}\right)} B\left(p_{i}, r\right)=c\left(s_{i}\right)^{\frac{n}{2}} \operatorname{Vol}_{g\left(t_{i}\right)} B\left(p_{i}, \tilde{r}\right)$, where $\tilde{r}$ might be a different radius as a matter of scaling and since $Q_{i} \leq \frac{C}{c\left(s_{i}\right)}$ (because the curvature of $g(t)$ is uniformly bounded), we get that:

$$
\operatorname{Vol}_{g\left(t_{i}\right)}(M) \geq \tilde{C} / C
$$

where $\tilde{C}$ and $C$ do not depend on $i$. Let $i \rightarrow \infty$ in the previous inequality to get a contradiction. Therefore, we have a uniform lower bound on volumes.

Remark 3.4. The assumptions of the Theorem 3.2 and the result of Claim 3.3 imply the uniform bounds on the curvature tensors, uniform upper bound on the diameters and uniform lower bounds on the volumes. Similarly, like in the case of unnormalized flow, uniform bounds on the curvatures gives us uniform bounds on all covariant derivatives, so by Hamilton's compactness theorem, for every sequence $t_{i} \nearrow \infty$ as $i \rightarrow \infty$, there exists a subsequence (call it again $\left.t_{i}\right)$, such that $\left(M, g\left(t_{i}+t\right)\right)$ converges to $(M, h(t))$, in the sense that there exist diffeomorphisms $\phi_{i}: M \rightarrow M$, so that $\phi_{i}^{*} g\left(t_{i}+t\right)$ converge uniformly together with their covariant derivatives to metrics $h(t)$ on compact subsets of $M \times[0, \infty)$. Moreover, $h(t)$ is a solution of a $\tau$-flow as well.

\subsection{Continuity of the minimizers for $\mathcal{W}$.}

We will recall a definition of Perelman's functional $\mathcal{W}=(4 \pi \tau)^{-\frac{n}{2}} \int_{M} e^{-f}[\tau$ $\left.\left(R+|\nabla f|^{2}\right)+f-n\right] d V$. The constraint on $f$ for this functional is $(4 \pi \tau)^{-\frac{n}{2}} \int e^{-f} d V=1$. Let $\mu(g, \tau)=\inf \mathcal{W}(g, f, \tau)$ under the constraint. This infinimum has been achieved by some smooth minimizer $f$. Perelman has also proved that for a fixed metric $g, \lim _{\tau \rightarrow 0} \mu(g, \tau)=0$ and $\mu(g, \tau)<0$ for a small value of $\tau>0$. 
In the case of a $\tau$-flow $g(t), \tau>0$ is being fixed in time, and by the monotonicity formula for $\mathcal{W}$, we have that $\mu(g(t), \tau)$ is increasing along the flow. Therefore, there exists $\lim _{t \rightarrow \infty} \mu(g(t), \tau)$.

Claim 3.5. $\lim _{t \rightarrow \infty} \mu(g(t), \tau)$ is finite.

Proof. Assume that $\lim _{t \rightarrow \infty} \mu(g(t), \tau)=\infty$. Then, $\forall i, \exists t_{i}$ s.t. $\mu\left(g\left(t_{i}\right), \tau\right) \geq$ $i$. There exists a subsequence (call it $t_{i}$ ) such that $\left(M, g_{i}\right)$ converges to $(M, h)$, for some metric $h$. By the proof Lemma 3.6 up to estimate, we get that $\mu\left(g\left(t_{i}\right), \tau\right)<\mu(h, \tau)+\epsilon$, for $i$ big enough. Letting $i \rightarrow \infty$, we get a contradiction.

Lemma 3.6. If $\left(M, g_{i}\right)$ tend to $(M, h)$ when $i \rightarrow \infty$, where $g_{i}=g\left(t_{i}\right)$ and $t_{i} \nearrow \infty$, then $\lim _{i \rightarrow \infty} \mu\left(g_{i}, \tau\right)=\mu(h, \tau)$.

Proof.

$$
\mu(h, \tau)=\int_{M}\left(\tau\left(|\nabla f|^{2}+R(h)\right)+f-n\right)(4 \pi \tau)^{-\frac{n}{2}} d V_{h} .
$$

Since, $\phi_{i}^{*} g_{i} \rightarrow h$ uniformly with their covariant derivatives, if $\epsilon>0$ is fixed, there exists some big $i_{0}$, so that for $i \geq i_{0}$

$$
\mu(h, \tau) \geq \int_{M}\left(\tau\left(|\nabla f|^{2}+R\left(\tilde{g}_{i}\right)\right)+f-n\right)(4 \pi \tau)^{-\frac{n}{2}} d V_{\tilde{g}_{i}}-\frac{\epsilon}{2},
$$

where $\tilde{g}_{i}=\phi^{*} g_{i}$. Change the variables in the above integral by diffeomorphism $\phi_{i}$.

$$
\mu(h, \tau) \geq \int_{M}\left(\tau\left(\left|\nabla_{i} f_{i}\right|^{2}+R\left(g_{i}\right)\right)+f_{i}-n\right)(4 \pi \tau)^{-\frac{n}{2}} d V_{g_{i}}-\frac{\epsilon}{2},
$$

where $f_{i}=\phi^{*} f$. Perturb a little bit $f_{i}$ to get $\tilde{f}_{i}$, by a quantity that tends to zero, so that $\int_{M} e^{-\tilde{f}_{i}}(4 \pi \tau)^{-\frac{n}{2}} d V_{g_{i}}=1$. Since our geometries are uniformly bounded, for big enough $i_{0}$, we will have

$$
\mu(h, \tau) \geq \mathcal{W}\left(g_{i}, \tilde{f}_{i}, \tau\right)-\epsilon \geq \mu\left(g_{i}, \tau\right)-\epsilon .
$$


Let $u_{i}=e^{-\frac{f_{i}}{2}}$. We have seen that minimizing $\mu\left(g_{i}, \tau\right)$ by $f_{i}$ is equivalent to minimizing the following expression in $u_{i}$ :

$$
\left.\int_{M} \tau\left(4\left|\nabla_{i} u_{i}\right|^{2}+R_{i} u_{i}^{2}\right)-2 u_{i}^{2} \ln u_{i}-n u_{i}^{2}\right)(4 \pi \tau)^{-\frac{n}{2}} d V_{g_{i}}
$$

The minimizer $u_{i}$ has to satisfy the following elliptic differential equation

$$
\tau\left(-4 \Delta_{i} u_{i}+R_{i} u_{i}\right)-2 u_{i} \ln u_{i}-n u_{i}=\mu_{i, \tau} u_{i}
$$

$\mu_{i, \tau}$ is uniformly bounded, since there is a finite $\lim _{t \rightarrow \infty} \mu(g(t), \tau)$. Now, we can easily get:

$$
\begin{gathered}
\int_{M} u_{i}^{2}(4 \pi \tau)^{-\frac{n}{2}} d V_{i} \leq C, \\
\tau \int_{M}\left|\nabla_{i} u_{i}\right|^{2}(4 \pi \tau)^{-\frac{n}{2}} d V_{i} \leq C,
\end{gathered}
$$

i.e. $u_{i} \in W^{1,2}$ with

$$
\left\|u_{i}\right\|_{W^{1,2}} \leq C \quad \forall i
$$

From (3.5), by standard regularity theory of partial differential equations and Sobolev embedding theorems, we get that $u_{i} \in W^{k, p}$ with uniformly bounded $W^{k, p}$ norms, where $p<\frac{2 n}{n-2}$, and therefore, with uniformly bounded $C^{2, \alpha}$ norms, i.e. $\left\|u_{i}\right\|_{C^{2, \alpha}} \leq C$. Furthermore,

$$
\begin{aligned}
\mu\left(g_{i}, \tau\right) & =\int_{M}\left(\tau\left(4\left|\nabla_{i} u_{i}\right|^{2}+R_{i} u_{i}^{2}\right)-2 u_{i}^{2} \ln u_{i}-n u_{i}^{2}\right)(4 \pi \tau)^{-\frac{n}{2}} d V_{i} \\
& \left.=\int_{M} \tau\left(\left|\tilde{\nabla} \tilde{u}_{i}\right|^{2} 4+\tilde{R}_{i} \tilde{u}_{i}{ }^{2}\right)-2 \tilde{u}_{i} \ln \tilde{u}_{i}-n \tilde{u}_{i}{ }^{2}\right)(4 \pi \tau)^{-\frac{n}{2}} d V_{\tilde{g}_{i}}(3.8)
\end{aligned}
$$

where $\tilde{u}_{i}=\phi_{i}^{*} u_{i}$. $\phi_{i}^{*} g_{i}$ is close to $h$ and therefore, for $i$ big enough, $\phi_{i}$ is almost an isometry, so $D_{j} \phi_{i}^{-1}$ can be uniformly bounded in terms of bounds on $g_{i}$ and $h, g_{i}$ can be bounded in terms of $h$. We cover $M$ with finitely many geodesic balls of fixed radius $\rho$ ( we can do it since, we have a uniform bound on the injectivity radii from below). We use local coordinates in each of the balls to get:

$$
\left|\tilde{\nabla}_{i} \tilde{u}_{i}\right|^{2}=\tilde{g}_{i}^{j k} D_{j}\left(u_{i} \circ \phi_{i}^{-1}\right) D_{k}\left(u_{i} \circ \phi_{i}^{-1}\right) .
$$




$$
\left|\tilde{\nabla} \tilde{u}_{i}\right|^{2}=\tilde{g}_{i}^{j k}\left(D_{j} u_{i}\right)\left(D_{k} u_{i}\right)\left(\phi_{i}^{-1}\right) D_{j} \phi_{i}^{-1} D_{k} \phi_{i}^{-1} .
$$

Now, we can easily conclude that we have a uniform bound on $\left|\tilde{\nabla} u_{i}\right|^{2}$. Since the integrand in (3.8) is uniformly bounded in $i$, and since $\tilde{g}_{i}$ uniformly converge with their covariant derivatives to $h$, we have that for $i$ large enough

$$
\mu\left(g_{i}, \tau\right) \geq \int_{M}\left(\tau\left(4\left|\nabla_{h} \tilde{u}_{i}\right|^{2}+R_{h} \tilde{u}_{i}^{2}\right)-2 \tilde{u}_{i} \ln \tilde{u}_{i}-n \tilde{u}_{i}^{2}\right)(4 \pi \tau)^{-\frac{n}{2}} d V_{h}-\epsilon .
$$

Since $l_{i}=\int_{M} \tilde{u}_{i}^{2}(4 \pi \tau)^{-\frac{n}{2}} d V_{h}$ is close to 1 when $i \rightarrow \infty$, taking $\bar{u}_{i}=\frac{\tilde{u}_{i}}{l_{i}}$ and using all the uniform bounds that we have got by now

$$
\mu\left(g_{i}, \tau\right) \geq \mathcal{W}\left(h, \bar{u}_{i}, \tau\right)-\epsilon \geq \mu(h, \tau)-\epsilon .
$$

By the previous inequality (for $i$ big enough) and by (3.4), we get

$$
\lim _{i \rightarrow \infty} \mu\left(g_{i}, \tau\right)=\mu(h, \tau)
$$

Following the notation from the previous lemma, by Arzela-Ascoli theorem, there exists a subsequence, $u_{i}$, so that it converges in $C^{2, \alpha}$ norm to some function $u$. We can also get the higher order uniform estimates on $u_{i}$ in a similar manner as in Lemma 3.6. Therefore, to show that a sequence of minimizers for $\mu\left(g_{i}, \tau\right)$ converges to a minimizer of $\mu(h, \tau)$ it is enough to show the following lemma.

Lemma 3.7. $\exists C>0$ so that $u_{i} \geq C>0 \quad \forall i$ and $\forall x \in M$

Proof. Assume that there exists a sequence $u_{i}$ and $p_{i} \in M$, such that $0<$ $u_{i}\left(p_{i}\right)<\frac{1}{2 i}$. $M$ is compact and therefore, there is a subsequence, $\left\{p_{i}\right\}$ converging to $p \in M$ when $i \rightarrow \infty$. $C^{2, \alpha}$ norms of $u_{i}$ are uniformly bounded in $i$ and therefore, $u_{i}(p)<u_{i}\left(p_{i}\right)+C \operatorname{dist}_{g_{i}}\left(p, p_{i}\right) \rightarrow 0$ as $i \rightarrow \infty$. Let $u$ be a limit of $\left\{u_{i}\right\}$ in $C^{2, \alpha}$ norm. Then $u(p)=0$. Take a geodesic ball $B(p, r)$. Let $f \in$ $C_{0}^{\infty}(M)$ be a $C^{\infty}$ function of $r$ alone, compactly supported in $B(p, r) \backslash\{p\}$.

$$
\int_{M}\left(\tau\left(\nabla u_{i} \nabla f+R_{i} u_{i} f\right)-2 u_{i} f \ln u_{i}-n u_{i} f-\mu\left(g_{i}, \tau\right) u_{i} f\right) d V_{i}=0 .
$$


For this $f$, letting $i \rightarrow \infty$, using the result of the previous lemma and the fact that the integrand in the previous integral is uniformly bounded in $i$, we get

$$
\int_{M}(\tau(\nabla u \nabla f+f u R(h))-2 u f \ln u-n u f-\mu(h, \tau) f u) d V_{h}=0 .
$$

Proceeding in the same manner as in [12], we can get that $u \equiv 0$ in some small ball around $p$. Using the connectedness argument, $u \equiv 0$ in $M$. On the other hand, $\int_{M} u_{i}^{2}(4 \pi \tau)^{-\frac{n}{2}} d V_{i}=1$ and letting $i \rightarrow \infty$, we get a contradiction.

If we write down the equations (3.5) for all $\left\{u_{i}\right\}$, letting $i \rightarrow \infty$, keeping in mind the previous lemma, we get

$$
\tau(-4 \Delta u+R(h)) u-2 u \ln u-n u=\mu(h, \tau) u,
$$

i.e. $u$ is the minimizer for $\mu(h, \tau)$.

So far, we have proved the following theorem

Theorem 3.8. If $\left(M, g_{i}\right) \rightarrow(M, h)$ as $i \rightarrow \infty$, then for a given $\tau>0$, if $\mu\left(g_{i}, \tau\right)=\mathcal{W}\left(g_{i}, f_{i}, \tau\right)$, then $f_{i} \rightarrow f$ in $C^{2, \alpha}$ norm, where $\mu(h, \tau)=$ $\mathcal{W}(h, f, \tau)$.

\subsection{Further estimates on the minimizers.}

In this subsection, we want to use the minimizers $f_{t}$ for $\mathcal{W}$ at different times to construct the functions $f_{t}(s)$ for $s \in[0, t]$. By using the parabolic regularity, we will be able to get the uniform estimates on $C^{k, \alpha}$ norms of $f_{t}(s)$. This will enable us to take a limit of these functions along the sequences. These limits are the functions that will turn out to be the potential functions that come into the equations describing the soliton type solutions arising in a limit.

For any $t$, we can find $f_{t}$ such that $\mathcal{W}\left(g(t), f_{t}, \tau\right)=\mu(g(t), \tau)$. If we flow $f_{t}$ backward, we will get functions $f_{t}(s)$ that satisfy

$$
\begin{aligned}
\frac{d f_{t}(s)}{d s} & =-R(s)-\Delta f_{t}(s)+\left|\nabla f_{t}(s)\right|^{2}+\frac{n}{2 \tau}, \\
f_{t}(t) & =f_{t} .
\end{aligned}
$$


We know that minimizing $\mathcal{W}$ in $f$ is equivalent to minimizing the corresponding functional in $\tilde{u}$, where $\tilde{u}_{t}=e^{-\frac{f_{t}}{2}}$. Let $u_{t}(s)=\tilde{u}_{t}^{2}(s)$. The equation for $u_{t}(s)$ is

$$
\begin{aligned}
\frac{d u_{t}}{d s} & =-\Delta u_{t}+\left(-\frac{n}{2 \tau}+R(s)\right) u_{t}(s), \\
u_{t}(t) & =u_{t} .
\end{aligned}
$$

By the monotonicity of $\mathcal{W}$ along the flow (3.1), we have that

$$
\mu(g(s), \tau) \leq \mathcal{W}\left(g(s), f_{t}(s), \tau\right) \leq \mathcal{W}\left(g(t), f_{t}, \tau\right)=\mu(g(t), \tau)
$$

First of all, there exists $\lim _{t \rightarrow \infty} \mu(g(t), \tau)$. It is finite, since for every sequence $t_{i} \rightarrow \infty$, there exists a subsequence such that $g\left(t_{i}\right) \rightarrow h(0)$ and by Lemma 3.6 from the previous section, we have that $\mu\left(g\left(t_{i}\right), \tau\right) \rightarrow \mu(h(0), \tau)$.

Instead of functional $\mathcal{W}\left(g(s), f_{t}(s), \tau\right)$, we can consider the equivalent functional which depends on $\tilde{u}_{t}(s)=e^{-f_{t}(s) / 2}$.

$$
\begin{array}{r}
\mathcal{W}\left(u_{t}(s)\right)=\int_{M}\left[\tau\left(4\left|\nabla \tilde{u}_{t}(s)\right|^{2}+R \tilde{u}_{t}(s)^{2}\right)-\tilde{u}_{t}(s)^{2} \log \tilde{u}_{t}(s)^{2}\right. \\
\left.-n \tilde{u}_{t}(s)^{2}\right](4 \pi \tau)^{-n / 2} d V
\end{array}
$$

where $\tilde{u}_{t}$ satisfy

$$
\tau\left(-4 \Delta \tilde{u}_{t}+R \tilde{u}_{t}\right)-2 \tilde{u}_{t} \ln \tilde{u}_{t}-n \tilde{u}_{t}=\mu(g(t), \tau) \tilde{u}_{t}
$$

since $f_{t}$ is a minimizer for $\mathcal{W}$. Since $\mu(g(t), \tau)$ is uniformly bounded, as in the previous section, we can get that $C^{2, \alpha}$ norms of $\tilde{u}_{t}$ are uniformly bounded. This implies that $C^{2, \alpha}$ norms of $u_{t}$ are uniformly bounded. Before we proceed with further discussion, notice the following.

Remark 3.9. $\int_{M}(4 \pi \tau)^{-\frac{n}{2}} e^{-f_{t}(s)} d V_{g(s)}=1$. This is a simple consequence of the fact that $\int_{M}(4 \pi \tau)^{-\frac{n}{2}} e^{-f_{t}} d V_{g(t)}=1$, since $f_{t}$ is a minimizer for $\mathcal{W}$ with respect to $g(t)$, and the following backward parabolic equation

$$
\frac{d}{d s} f_{t}(s)=-\Delta f_{t}(s)+\left|\nabla f_{t}(s)\right|^{2}-R+\frac{n}{2 \tau} .
$$


Namely,

$$
\begin{aligned}
\frac{d}{d s}\left(\int_{M} e^{-f_{t}(s)} d V_{g(s)}\right) & \\
& =\int_{M} e^{-f_{t}(s)}\left(\Delta f_{t}(s)-\left|\nabla f_{t}(s)\right|^{2}+R-\frac{n}{2 \tau}-R+\frac{n}{2 \tau}\right) d V_{g(s)} \\
& =\int_{M} \Delta\left(e^{-f_{t}(s)} d V_{g(s)}\right)=0
\end{aligned}
$$

Since $\log$ is a concave function and $\tilde{u}_{t}(s)^{2}(4 \pi \tau)^{-n / 2} d V$ is a probability measure, we have by Jensen and Sobolev inequalities

$$
\begin{aligned}
& \int_{M} \tilde{u}_{t}(s)^{2} \log \tilde{u}_{t}(s)^{2}= \\
&(4 \pi \tau)^{-n / 2} d V= \frac{n-2}{2} \int_{M} \tilde{u}_{t}(s)^{2} \log \tilde{u}_{t}(s)^{4 /(n-2)}(4 \pi \tau)^{-n / 2} d V \\
& \leq \frac{n-2}{2} \log \int_{M} \tilde{u}_{t}(s)^{2 n /(n-2)}(4 \pi \tau)^{-n / 2} d V \\
& \leq \frac{n-2}{2} \log \left[C \int_{M}\left(\left|\nabla \tilde{u}_{t}(s)\right|^{2}+\tilde{u}_{t}(s)^{2}\right) d V\right]^{(n-2) / n} \\
&+\frac{n-2}{2} \log (4 \pi \tau)^{-n / 2} \\
&= \frac{n}{2} \log C \int_{M} \tau\left(\left|\nabla \tilde{u}_{t}(s)\right|^{2}+\tilde{u}_{t}(s)^{2}\right)(4 \pi \tau)^{-n / 2} d V .
\end{aligned}
$$

This inequality shows that

$$
\tau \int_{M}\left|\nabla \tilde{u}_{t}(s)\right|^{2}(4 \pi \tau)^{-n / 2} d V \leq C .
$$

The constant $C$ does not depend either on $t$ or $s \in[0, t]$. To conclude, we have the following estimates

$$
\begin{gathered}
\int_{M}\left|\tilde{u}_{t}(s)\right|^{2}(4 \pi \tau)^{-\frac{n}{2}} d V_{s} \leq C_{1} \\
\tau(4 \pi \tau)^{-\frac{n}{2}} \int_{M}\left|\nabla_{s} \tilde{u}_{t}(s)\right|^{2} d V_{s} \leq C_{2},
\end{gathered}
$$

that is, we have that $\left|\tilde{u}_{t}\right|_{W_{1,2}} \leq C$ for a uniform constant $C$.

Take a sequence $t_{i} \rightarrow \infty$. There exists a subsequence such that $g\left(t_{i}+t\right) \rightarrow$ $h(t)$ when $i \rightarrow \infty$, where $h(t)$ is a Ricci flow on $M$. This follows from Hamilton's compactness theorem ([8]). Fix $A>0 . f_{t}$ will be a minimizer for $\mathcal{W}$ with respect to $g(t)$, which we flow backward, for every $t$. Let $s \in[0, A]$. 
Lemma 3.10. For every $A>0$, there exists $\delta=\delta(A)>0$ such that $u_{t+A}(t+s) \geq \delta>0$ for all $t$ and all $s \in[0, A]$.

Proof. Assume that the statement of the lemma is not true. In that case, there would exist a sequence $s_{i}$ such that $\min _{M} u_{s_{i}+A}\left(s_{i}+a_{i}\right) \rightarrow 0$ as $i \rightarrow \infty$, for some $a_{i} \in[0, A]$. Consider the equation

$$
\begin{aligned}
\frac{d}{d t} u_{s_{i}+A}\left(s_{i}+t\right) & =-\Delta u_{s_{i}+A}\left(s_{i}+t\right)+\left(R-\frac{n}{2 \tau}\right) u_{s_{i}+A}\left(s_{i}+t\right), \\
u_{s_{i}+A}\left(s_{i}+A\right) & =u_{s_{i}+A}
\end{aligned}
$$

for $t \in[0, A]$. Let $\hat{u}_{i}\left(s_{i}+t\right)=\min _{M} u_{s_{i}+A}\left(s_{i}+t\right)$. Then $\Delta \hat{u}_{s_{i}+A}\left(s_{i}+t\right) \geq 0$ and

$$
\frac{d}{d t} \hat{u}_{i}\left(s_{i}+t\right) \leq C \hat{u}_{i}\left(s_{i}+t\right)
$$

where $C$ is a uniform constant. If we integrate it with respect to $t$, we get

$$
\hat{u}_{i}\left(s_{i}+A\right) \leq e^{C A} \hat{u}_{i}\left(s_{i}+t\right) .
$$

Since $\hat{u}_{i}\left(s_{i}+A\right)=\min _{M} u_{s_{i}+A}$ and since by Lemma 3.7, we know that there exists a constant $\delta$ such that $u_{s_{i}+A} \geq \delta>0$, we have that $u_{s_{i}+A}\left(s_{i}+t\right) \geq$ $\delta(A)>0$ for all $i$ and all $t \in[0, A]$. This contradicts our assumption that $\hat{u}_{i}\left(s_{i}+a_{i}\right) \rightarrow 0$ as $i \rightarrow \infty$.

Lemma 3.11. For every $A>0$, there exists $C(A)$ such that

1. $\int_{M} u_{t}(s)^{2} d V_{g(s)} \leq C(A)$.

2. $\int_{M}\left|\nabla u_{t}(s)\right|^{2} d V_{g(s)} \leq C(A)$,

for all $t \geq A, s \in[t-A, t]$.

Proof. We will consider the equation

$$
\begin{aligned}
\frac{d}{d s} u_{t}(s) & =-\Delta u_{t}(s)+\left(R-\frac{n}{2 \tau}\right) u_{t}(s) \\
u_{t}(t) & =u_{t},
\end{aligned}
$$


where $u_{t}=e^{-f_{t}}$ and $f_{t}$ is a minimizer for $\mathcal{W}$ with respect to metric $g(t)$. Let $\hat{u}_{t}(s)=\max _{M} u_{t}(s)$. Then

$$
\frac{d}{d s} \hat{u}_{t}(s) \geq-C \hat{u}_{t}(s)
$$

where $C>0$ is a uniform constant that does not depend either on $s$ or $t$, but on the uniform bounds on geometries $g(t)$. If we integrate it with respect to $s$, we get

$$
\hat{u}_{t}=\hat{u}_{t}(t) \geq e^{-C A} \hat{u}_{t}(s)
$$

for any $s \in[t-A, t]$. On the other hand, we have already proved in the previous section that $C^{2, \alpha}$ norms of $u_{t}$ are uniformly bounded in $t \in[0, \infty)$. Therefore, we get that $0 \leq u_{t}(s) \leq C(A)$ on $M$ for all $t \in[A, \infty)$ and all $s \in[t-A, t]$. Now we immediately get part 1 of our claim. For part 2, notice that

$$
\int_{M}\left|\nabla u_{t}(s)\right|^{2} d V_{g(s)}=4 \int_{M} u_{t}(s)\left|\nabla \tilde{u}_{t}(s)\right|^{2} d V_{g(s)} \leq \tilde{C}(A),
$$

since $\int_{M}\left|\nabla \tilde{u}_{t}(s)\right|^{2}$ is uniformly bounded for all $t \geq A$ and $s \in[t-A, t]$.

The previous two lemmas tell us that in order to find the uniform estimates on $f_{t_{i}+A}\left(t_{i}+s\right)$ for $s \in[0, A]$, it is enough to find the uniform $C^{k, \alpha}$ estimates on $u_{t_{i}+A}\left(t_{i}+s\right)$. Our main goal in this section is to prove the following theorem.

Theorem 3.12. Under the assumptions of the main theorem, with the notations as above, for every $A>0$, there exists a uniform constant $C$, depending on $A$ such that $\left|u_{t}(s)\right|_{C^{2, \alpha}} \leq C$ for all $t \geq A, \forall s \in[t-A, t]$.

Proof. Consider the equation

$$
\frac{d}{d s} u_{t}(s)=-\Delta u_{t}(s)+\left(R(s)-\frac{n}{2 \tau}\right) u_{t}(s)
$$


for $t \in[A, \infty)$ and $s \in[t-A, t]$. All our further estimates will depend on $A$. We will use $C$ to denote different absolute constants that depend on $A$ and the uniform bounds on our geometries $g(t)$. Denote by $h_{1}=\left(h_{1}\right)_{t}(s)=$ $\left(-\frac{n}{2 \tau}+R(s)\right) u_{t}(s)$. Omit the subscript $t$.

$$
\begin{gathered}
\frac{d}{d s} u+\Delta u=h_{1} . \\
\int_{M} h_{1}^{2}=\int_{M}\left(\frac{d}{d s} u\right)^{2}+2 \int_{M} \frac{d}{d s} u \Delta u+\int_{M}(\Delta u)^{2},
\end{gathered}
$$

where we should keep in mind that the metric depends on $s$.

$$
\begin{aligned}
\int_{M} \frac{d}{d s} u \Delta u= & -\int_{M} g^{i j} \nabla_{i}\left(\frac{d}{d s} u\right) \nabla_{j} u d V_{s} \\
= & -\frac{1}{2} \frac{d}{d s} \int_{M}|\nabla u|^{2} d V_{s}-\int_{M}|\nabla u|^{2}\left(\frac{n}{2 \tau}-R\right) d V_{s} \\
& +\int_{M} g^{p i} g^{q j} D_{i} u D_{j} u\left(2 R_{p q}-\frac{1}{2 \tau} g_{p q}\right) d V_{s},
\end{aligned}
$$

where the second term on the right-hand side of (3.12) comes from taking the derivative of the volume element and the third term appears from taking the derivative of $g^{i j}$. Denote the former one by $J_{1}$ and the latter one by $J_{2}$.

$$
\begin{aligned}
\int_{M}(\Delta u)^{2} & =\int_{M} g^{i j} D_{i} D_{j} u g^{k l} D_{k} D_{l} u \\
& =-\int_{M} g^{i j} g^{k l} D_{j} u D_{i} D_{k} D_{l} u \\
& =-\int_{M} g^{i j} g^{k l} D_{j} u D_{k} D_{i} D_{l} u+\int_{M} g^{i j} g^{k l} D_{j} u R_{i k s}^{l} D_{s} u \\
& =I+\int_{M} g^{i j} g^{k l} D_{k} D_{j} u D_{i} D_{l} u \\
& =I+\int_{M}\left|\nabla^{2} u\right|^{2},
\end{aligned}
$$

where $I=\int_{M} g^{i j} g^{k l} D_{j} u R_{i k s}^{l} D_{s} u$. Let $l \in(t-A, t)$ where $A>0$. Integrating, the equation (3.11) in $s$, from $l$ to $t$ gives

$$
\begin{array}{r}
\int_{l}^{t}\left(\int_{M}\left(\frac{d}{d s} u\right)^{2} d V_{s}\right) d s+\left.\int_{M}|\nabla u|^{2} d V_{s}\right|_{s=l}+\int_{l}^{t} \int_{M}\left|\nabla^{2} u\right|^{2} d V_{s} d s \\
=\int_{l}^{t} \int_{M} h_{1}^{2}+\left.\int_{M}|\nabla u|^{2} d V_{s}\right|_{s=t}+\int_{l}^{t}\left(2 J_{1}+2 J_{2}+I\right) .
\end{array}
$$




$$
\int_{l}^{t} J_{1} \leq A C \sup _{s \in(t-A, t)} \int_{M}|\nabla u|^{2} d V_{s} \leq \tilde{C}
$$

for every $t$. Similarly, we get estimates for $J_{2}$ and $I$. From all these estimates, we can conclude the following

$$
\begin{aligned}
& \int_{t-A}^{t} \int_{M}\left(\frac{d}{d s} u_{t}(s)\right)^{2} d V_{s} d s \leq C . \\
& \int_{t-A}^{t} \int_{M}\left|\nabla^{2} u_{t}(s)\right|^{2} d V_{s} d s \leq C . \\
& \sup _{s \in(t-A, t)} \int_{M}|\nabla u|^{2} d V_{s} \leq C,
\end{aligned}
$$

where $C=C(A)$. Let $\tilde{u}_{t}=\frac{d}{d s} u_{t}(s)$ (we will not confuse this $\tilde{u}_{t}$ with one defined at the beginning of this section). Omit the subscript $t$.

$$
\frac{d}{d s} \tilde{u}=-D_{s} \Delta_{s} u+\frac{d}{d s}\left[\left(R-\frac{n}{2 \tau}\right) u\right] .
$$

Multiply the equation by $\tilde{u}$ and integrate it along $M$.

$$
\begin{aligned}
\frac{1}{2} \frac{d}{d s} \int_{M}\left|\frac{d}{d s} u\right|^{2} d V_{s}= & -\int_{M} \frac{d}{d s}\left(g(s)^{i j} D_{i} D_{j} u\right) \tilde{u}+\int_{M}\left(\frac{d}{d s}\left(R-\frac{n}{2 \tau}\right)\right) u \tilde{u} \\
& +\frac{1}{2} \int_{M}\left(R-\frac{n}{2 \tau}\right)\left|\frac{d}{d s} u\right|^{2} d V_{s} \\
= & 2 \int_{M}\left(-R_{p q}+\frac{1}{2 \tau} g_{p q}\right) g^{p i}(s) g^{q j}(s) D_{i} D_{j} u \tilde{u} \\
& -\int_{M} g(s)^{i j} D_{i} D_{j}\left(\frac{d}{d s} u\right) \tilde{u} \\
& +\int_{M}\left(\frac{d}{d s}\left(R-\frac{n}{2 \tau}\right)\right) u \tilde{u} \int_{M} g^{j k}\left(\frac{d}{d t} \Gamma_{i j}^{k}\right) \frac{\partial u}{\partial x_{k}} \tilde{u} \\
& +\frac{1}{2} \int_{M}\left(R-\frac{n}{2 \tau}\right)\left|\frac{d}{d s} u\right|^{2} d V_{s} .
\end{aligned}
$$

Since $\int_{M} g(s)^{i j} D_{i} D_{j} \frac{d}{d s} u \tilde{u}=-\int_{M}\left|\nabla_{s}\left(\frac{d}{d s} u\right)\right|^{2}$ and since we are on the Ricci flow, metrics $g(s)$ are uniformly bounded, after applying Cauchy-Schwartz 
inequality and using the uniform boundedness of the curvature operator, we get

$$
\begin{aligned}
\int_{t-A}^{t} \int_{M}\left|\nabla\left(\frac{d}{d s} u\right)\right|^{2} d V_{s} d s+ & \sup _{s \in(}(t-A, t) \\
\leq & C \int_{t-A}^{t}\left|\frac{d}{d s} u\right|^{2} \\
& +C \int_{t-A}^{t} \int_{M}\left|\frac{d}{d s} u\right|^{2} d V_{s} d s \\
& +\int_{M}\left|\frac{d}{d s} u\right|^{2} d V_{s} d s
\end{aligned}
$$

$\left.\int_{M}\left|\frac{d}{d s} u(s)\right|^{2} d V_{s}\right|_{s=t} \leq C\left(\int_{M}\left|\Delta u_{t}\right|^{2}+\int_{M} h_{1}(t)^{2}\right)$ where $h_{1}(s)=\left(\frac{n}{2 \tau}-\right.$ $R(s)) u(s)$. Since $u_{t}=e^{-f_{t}}$, where $f_{t}$ are the minimizers for $\mathcal{W}$, like in the previous section, we can conclude that $u_{t} \in W^{k, p}$, with uniform bounds on $W^{k, p}$ norms (these bounds depend on $k$ ) and therefore, $\int_{M}\left|\frac{d}{d s} u(s) d V_{s}\right|_{s=t}$ are uniformly bounded in $t$. This estimate together with estimates (3.13) and (3.14) gives that

$$
\begin{gathered}
\int_{t-A}^{t} \int_{M}\left|\nabla\left(\frac{d}{d s} u\right)\right|^{2} d V_{s} d s \leq C . \\
\sup _{s \in(t-A, t)} \int_{M}\left|\frac{d}{d s} u\right|^{2} \leq C .
\end{gathered}
$$

If $\tilde{u}=\frac{d}{d s} u$ and $\tilde{h}=\frac{d}{d s} h_{1}$ then:

$$
\begin{aligned}
\frac{d}{d s} \tilde{u}=- & D_{s} \Delta u+\tilde{h} . \\
D_{s} \Delta u=\frac{d}{d s}\left(g(s)^{i j} D_{i} D_{j} u\right)= & g(s)^{i p} g(s)^{j q}\left(\frac{1}{\tau} g_{p q}-2 R_{p q}\right) D_{i} D_{j} u \\
& +g(s)^{i j} D_{i} D_{j} \tilde{u} \\
& +g(s)^{i j} \frac{d}{d s}\left(\Gamma_{i j}^{k}\right) D_{k} u . \\
H=\tilde{h}-g^{i p} g^{j q}\left(\frac{1}{\tau} g_{p q}-2 R_{p q}\right) D_{i} D_{j} u-g(s)^{i j} \frac{d}{d s}\left(\Gamma_{i j}^{k}\right) D_{k} u &
\end{aligned}
$$


All the estimates that we have got so far tell that $\int_{t-A}^{t} \int_{M} H^{2}$ is uniformly bounded in $t$. The analogous estimates to the estimates (3.13), (3.14) and (3.15) for $u$, we can get for $\frac{d}{d s} u$ (by using the evolution equation for $\frac{d}{d s} u$ and all the estimates that we have got so far by analyzing the evolution equation for $u$ ).

$$
\begin{gathered}
\int_{t-A}^{t} \int_{M}\left|\nabla^{2}\left(\frac{d}{d s} u\right)\right|^{2} d V_{s} d s \leq C . \\
\int_{t-A}^{t} \int_{M}\left(\frac{d^{2}}{d s^{2}} u\right)^{2} d V_{s} d s \leq C . \\
\sup _{s \in(t-A, t)} \int_{M}\left|\nabla\left(\frac{d}{d s} u\right)\right|^{2} d V_{s} \leq C .
\end{gathered}
$$

To obtain these estimates, we have used the fact that

$$
\left.\int_{M}\left|\nabla \frac{d}{d s} u\right|^{2} d V_{g(s)}\right|_{s=t} \leq C\left(\int_{M}\left|\nabla \Delta u_{t}\right|^{2}+\int_{M}\left|\nabla\left(R-\frac{n}{2 \tau}\right) u_{t}\right|^{2},\right.
$$

where the right-hand side is uniformly bounded in $t$, since $u_{t}=e^{-f_{t}}$ and $f_{t}$ are the minimizers for $\mathcal{W}$.

By standard regularity theory, considering $\Delta u_{t}(s)=-\frac{d}{d s} u_{t}(s)+\left(h_{1}\right)_{t}(s)$ as an elliptic equation whose right-hand side has uniformly bounded $W^{1,2}$ norms for $s \in(t-A, t)$ and all $t \geq A$, we have that $\left|u_{t}(s)\right|_{W^{3,2}} \leq C$, for a uniform constant $C$ that depends on $A$. Take a derivative in $s$ of the equation $\frac{d}{d s} \tilde{u}=-\Delta \tilde{u}+H$, with $\tilde{u}=\frac{d}{d s} u$. Denote by $\bar{u}=\frac{d}{d s} \tilde{u}$. By using the estimates that we have got for $\tilde{u}$, it is easy to conclude that $\bar{u}$ satisfies the equation

$$
\frac{d}{d s} \bar{u}=-\Delta \bar{u}+H_{1}
$$

where

$$
H_{1}=\frac{d}{d s} H+g^{i p} g^{j q}\left(-2 R_{p q}+\frac{1}{\tau}\right) D_{i} D_{j} \tilde{u}+g(s)^{i j} \frac{d}{d s}\left(\Gamma_{i j}^{k}\right) D_{k} \tilde{u}
$$

and $\int_{t-A}^{t} \int_{M} H_{1}^{2} d V_{g(s)} d s$ is uniformly bounded in $t$. As in the case of the previous estimates, we can conclude that 


$$
\begin{gathered}
\sup _{s \in(t-A, t)} \int_{M}\left|\frac{d}{d s} \tilde{u}\right|^{2} d V_{s} \leq C, \\
\sup _{s \in(t-A, t)} \int_{M}\left|\nabla\left(\frac{d}{d s} \tilde{u}\right)\right|^{2} d V_{s} \leq C .
\end{gathered}
$$

By regularity theory applied to the equation $\Delta \tilde{u}=-\frac{d}{d s} \tilde{u}+H$, we can get that $\frac{d}{d s} u_{t}(s)$ has uniformly bounded $W^{3,2}$ norms. If we go back to the parabolic equation for $u_{t}(s)$, we can get that $\left|u_{t}(s)\right|_{W^{5,2}} \leq C$ for all $t \geq A$ and all $s \in(t-A, t)$. Continuing this process by taking more and more derivatives in $t$ of our original parabolic equation, we can conclude that $W^{p, 2}$ norms of $u_{t}(s)$ are uniformly bounded for every $p$, by the constants that depend on $A$ and $p$. Sobolev embedding theorem now gives that all $C^{k, \alpha}$ norms of $u_{t}(s)$ are uniformly bounded for all $t>A$ and all $s \in[t-A, t]$, by constants that depend on $A$ and $k$.

Combining Theorem 3.12 and Lemma 3.10, we get that for every $A$ there, exist constants $C_{k}=C(k, A)$ such that $\left|f_{t}(s)\right|_{C^{k, \alpha}} \leq C_{k}$, for all $t \geq A$ and all $s \in[t-A, t]$.

\subsection{Ricci soliton in the limit.}

In this subsection, we want to finish the proof of Theorem 3.2.

We have uniform curvature and diameter bounds for our flow $g(t)$. We have already proved that we also have a volume non-collapsing condition along the flow, for all times $t \geq 0$. This gives a uniform lower bound on the injectivity radii. Hamilton's compactness theorem (modified to the case of our flow) gives that for every sequence $t_{i} \rightarrow \infty$, there exists a subsequence so that $g\left(t_{i}+t\right) \rightarrow h(t)$ uniformly on compact subsets of $M \times[0, \infty)$ and that $h(t)$ is a solution to the Ricci flow (3.1). We will show below that for each $t$, $h(t)$ satisfies actually a Ricci soliton equation with the Hessian of function $f_{h}(t)$ involved, where $f_{h}(t)$ is a smooth one parameter family of functions. We will now see how we get the functions $f_{h}(t)$, using the estimates on $f_{t}(s)$ from the previous subsection and Perelman's monotonicity formula.

Take any $t$ and let $f_{t}$ be a function so that $\mu(g(t), \tau)=\mathcal{W}\left(g(t), f_{t}, \tau\right)$. Flow $f_{t}$ backward. Fix $A>0$. Then: 


$$
\begin{gathered}
I(t)=\mathcal{W}\left(g(t+A), f_{t+A}, \tau\right)-\mathcal{W}\left(g(t), f_{t+A}(t), \tau\right) \\
\leq \mu(g(t+A), \tau)-\mu(g(t), \tau) \rightarrow 0(t \rightarrow \infty) . \\
0 \leq I(t)=\int_{0}^{A} \frac{d}{d u} W\left(g(t+s), f_{t+A}(t+s), \tau\right) d s \rightarrow 0,
\end{gathered}
$$

as $t \rightarrow \infty$. We will consider $u_{t_{i}+A}\left(t_{i}+s\right)$ where $s \in[0, A]$. We will divide the proof of the theorem in a few steps.

Step 3.13. $\forall A>0, \lim _{i \rightarrow \infty} \frac{d}{d u} W\left(g\left(s+t_{i}\right), f_{t_{i}+A}\left(s+t_{i}\right), \tau\right)=0$ for almost all $s \in[0, A]$.

Proof. $I\left(t_{i}\right) \rightarrow 0$ by Claim 3.5. On the other hand

$$
\begin{aligned}
I\left(t_{i}\right) & =\mathcal{W}\left(g\left(t_{i}+A\right), f_{t_{i}+A}, \tau\right)-\mathcal{W}\left(g\left(t_{i}\right), f_{t_{i}+A}\left(t_{i}\right), \tau\right) \\
& =\int_{0}^{A} \frac{d}{d u} W\left(g\left(t_{i}+s\right), f_{t_{i}+A}\left(t_{i}+s\right), \tau\right) d s
\end{aligned}
$$

and

$$
\begin{aligned}
\lim _{i \rightarrow \infty} I\left(t_{i}\right) & =\lim _{i \rightarrow \infty} \int_{0}^{A} \frac{d}{d u} W\left(g\left(t_{i}+s\right), f_{t_{i}+A}\left(t_{i}+s\right), \tau\right) d s \\
& \geq \int_{0}^{A} \liminf _{i \rightarrow \infty} \frac{d}{d u} W\left(g\left(t_{i}+s\right), f_{t_{i}+A}\left(t_{i}+s\right), \tau\right) d s
\end{aligned}
$$

by Fatou's Lemma. Since by Perelman's monotonicity formula $\frac{d}{d u} W\left(g\left(t_{i}+s\right), f_{t_{i}+A}\left(t_{i}+s\right), \tau\right) \geq 0$, we have that $\lim _{i \rightarrow \infty} \frac{d}{d u} W\left(g\left(t_{i}+s\right), f_{t_{i}+}\left(t_{i}+s\right), \tau\right)=0$ for almost all $s \in[0, A]$, for

$$
\int_{0}^{A} \lim _{i \rightarrow \infty} \frac{d}{d u} W\left(g\left(t_{i}+s\right), f_{t_{i}+A}\left(t_{i}+s\right), \tau\right) d s \leq \lim _{i \rightarrow \infty} I\left(t_{i}\right),
$$

by Fatuous lemma.

Step 3.14. $\left|\tilde{u}_{t}(s)\right|_{C^{2, \alpha}} \leq C, \forall t$, where $\tilde{u}_{t}(s)=\frac{d}{d s} u_{t}(s)$.

Proof. Following the notation of the previous subsection, we get that:

$$
\frac{d}{d s} \tilde{u}_{t}(s)=-\Delta \tilde{u}_{t}(s)+H_{t}(s)
$$


where $H_{t}(s)=\frac{d}{d s} h_{t}(s)+g^{i p} g^{j q}\left(\frac{1}{\tau} g_{p q}-2 R_{p q}\right) D_{i} D_{j} u+g^{i j} \frac{d}{d s}\left(\Gamma_{i j}^{k}\right) D_{k} u$.

$$
\tilde{u}_{t}(t)=\frac{d}{d s} u_{t}(s)=-\Delta u_{t}+\left(-\frac{n}{2 \tau}+R\right) u_{t} .
$$

In the previous subsection, we have proved that there exists a uniform lower and an upper bound on $u_{t}(s)$ and that $\left|u_{t}(s)\right|_{W^{3, p}} \leq C(p, A)$ for all $t \geq A$ and all $s \in[t-A, t]$. Similarly, we can get that $\left|u_{t}(s)\right|_{W^{k, p}} \leq C(k, p, A)$ and therefore $\left|\tilde{u}_{t}(s)\right|_{W^{k-2, p}} \leq C(k, p, A), \forall t \geq A$ and all $s \in[t-A, t]$. We can get that $\left|\tilde{u}_{t}(s)\right|_{C^{2, \alpha}} \leq C$, for all $t \geq A$ and $\forall s \in[t-A, t]$. We can extend this to all higher order time derivatives of $u_{t}(s)$.

Step 3.15. For every $A>0$, there exists a subsequence $t_{i}$, so that the limit metric $h(s)$ of a sequence $g\left(t_{i}+s\right)$ is a Ricci soliton for $s \in[0, A]$.

Proof. By step 3.13, we have that

$$
\lim _{i \rightarrow \infty} R_{j k}\left(t_{i}+s\right)+\nabla_{j} \nabla_{k} f_{t_{i}+A}\left(t_{i}+s\right)-\frac{1}{2 \tau} g_{j k}\left(t_{i}+s\right)=0,
$$

for almost all $s \in[0, A]$ and almost all $x \in M$, since

$$
\begin{aligned}
& \frac{d}{d s} \mathcal{W}\left(g\left(t_{i}+s\right), f_{t_{i}+A}\left(t_{i}+s\right), \tau\right) \\
& \quad=(4 \pi \tau)^{-\frac{n}{2}} \int_{M} 2 \tau\left|R_{j k}+\nabla_{j} f_{t_{i}+A} \nabla_{k} f_{t_{i}+A}-\frac{1}{2 \tau} g_{j k}\right|^{2} d V_{g\left(t_{i}+s\right)} .
\end{aligned}
$$

By Lemma 3.10 and Theorem 3.12, we have that $0<C_{1} \leq\left|u_{t_{i}+A}\left(s+t_{i}\right)\right| \leq$ $C_{2}$ for all $i \geq i_{0}$ and all $s \in[0, A]$, for some constants $C_{1}$ and $C_{2}$ that depend on $A$. By step 3.14 and Theorem 3.12, we can find a subsequence, say $\left\{t_{i}\right\}$ such that $f_{t_{i}+A}\left(t_{i}+s\right)$ converges in $C^{2, \alpha}$ norm to $\tilde{f}_{A}(s)$ for all $s \in[0, A]$ and all $x \in M$. More precisely, for a countable dense subset $\left\{s_{j}\right\}$ of $[0, A]$ there exists a subsequence so that $f_{t_{i}+A}\left(t_{i}+s_{j}\right)$ converges in $C^{2, \alpha}$ norm to $\tilde{f}_{A}\left(s_{j}\right)$ on $M$. For any $s \in[0, A]$ there exists a subsequence $t_{i_{k}}$ so that $f_{t_{i_{k}}+A}\left(t_{i_{k}}+s\right)$ converges to $\tilde{f}_{A}(s)$ in $C^{2, \alpha}$ norm. We want to show that actually $f_{t_{i}+A}\left(t_{i}+s\right) \stackrel{C^{2, \alpha}}{\rightarrow} \tilde{f}_{A}(s)$. For that we use the fact that $\frac{d}{d s} f_{t_{i}+A}\left(t_{i}+s\right)$ is uniformly bounded in $C^{2, \alpha}$ norm, and therefore 


$$
\left|\tilde{f}_{A}(s)-\tilde{f}_{A}\left(s_{0}\right)\right|_{C^{2, \alpha}}<\epsilon,
$$

for some small $\epsilon>0$ and some $s_{0} \in\left\{s_{j}\right\}$ that is sufficiently close to $s$. We also have

$$
\left|\tilde{f}_{A}\left(s_{0}\right)-f_{t_{i}+A}\left(t_{i}+s_{0}\right)\right|_{C^{2, \alpha}}<\epsilon
$$

for $i \geq i_{0}$ and

$$
\left|f_{t_{i}+A}\left(t_{i}+s\right)-f_{t_{i}+A}\left(t_{i}+s_{0}\right)\right|_{C^{2, \alpha}}<\epsilon,
$$

since $\left|\frac{d}{d s} f_{t_{i}+A}\left(t_{i}+s\right)\right|_{C^{2, \alpha}} \leq C(A)$, for all $i \geq i_{0}$ and all $s \in[0, A]$. By triangle inequality, we now get that for every $\epsilon>0$ there exists $i_{0}$ so that

$$
\left|\tilde{f}_{A}(s)-f_{t_{i}+A}\left(t_{i}+s\right)\right|_{C^{2, \alpha}}<3 \epsilon,
$$

for all $i \geq i_{0}$ and all $s \in[0, A]$.

$f_{t_{i}+A}\left(t_{i}+s\right)$ converges in $C^{2, \alpha}$ norm on $M$ to $\tilde{f}_{A}(s)$, for all $s \in[0, A]$. Finally, we get that

$$
R_{j k}+\nabla_{j} \nabla_{k} \tilde{f}_{A}(s)-\frac{1}{2 \tau} h_{j k}(s)=0
$$

for all $s \in[0, A]$, and for almost all $x \in M$. Because of the continuity, it will hold for all $x \in M$. Since $h(s)$ is a Ricci flow, all covariant derivatives of $h$ and the covariant derivatives of a curvature operator are uniformly bounded, and therefore $\left|\nabla^{p} \tilde{f}_{A}(s)\right| \leq C(p), \forall s \in[0, A]$ and all $p \geq 2$. Also we have that $\left|\frac{d^{p}}{d s^{k}} \nabla^{p} \tilde{f}_{A}(s)\right| \leq C(p, k)$ where $C(p, k)$ does not depend on $A$, for $p \geq 2$.

Step 3.16. We can glue all the functions $\tilde{f}_{A}$ that we get for different values of $A$, to get a function $f_{h}(s)$ defined on $M \times[0, \infty)$, which defines our metric $h(s)$ as a soliton type solution for all times $s \geq 0$.

Proof. Take any increasing sequence $A_{j} \rightarrow \infty$. For every $A_{j}$, by the previous step, we can extract a subsequence $t_{i}$ so that $f_{t_{i}+A_{j}}\left(t_{i}+s\right) \stackrel{C^{2, \alpha}}{\rightarrow} \tilde{f}_{A_{j}}(s)$ for all $s \in\left[0, A_{j}\right]$. Diagonalization procedure gives a subsequence so that 
$f_{t_{i}+A_{j}}(s) \stackrel{C^{2, \alpha}}{\rightarrow} \tilde{f}_{A_{j}}(s)$ for all $j$ and all $s \in\left[0, A_{j}\right]$. For this subsequence $t_{i}$ we have that $g\left(t_{i}+t\right) \rightarrow h(t)$, uniformly on compact subsets of $M \times[0, \infty)$. Compare the functions $\tilde{f}_{A_{j}}$ and $\tilde{f}_{A_{k}}$ for $j<k$, on the interval $\left[0, A_{j}\right]$. We know that they both satisfy

$$
\Delta_{h(s)} \tilde{f}_{A_{r}}+R(h(s))-\frac{n}{2 \tau}=0
$$

and therefore, $\Delta_{h(s)}\left(\tilde{f}_{A_{j}}-\tilde{f}_{A_{k}}\right)=0$. Since $M$ is compact, this implies that $\tilde{f}_{A_{k}}(s)=\tilde{f}_{A_{j}}(s)+c_{A_{j}}^{A_{k}}(s)$, for $s \in\left[0, A_{j}\right]$, where $c_{A_{j}}^{A_{k}}(s)$ is a constant function for every $s \in\left[0, A_{j}\right]$. On the other hand, because of the integral normalization condition, we have

$$
\begin{gathered}
(4 \pi \tau)^{-\frac{n}{2}} \int_{M} e^{-\tilde{f}_{A_{j}}(s)} d V_{h(s)}=1 \\
(4 \pi \tau)^{-\frac{n}{2}} \int_{M} e^{-\tilde{f}_{A_{k}}(s)} d V_{h(s)}=1=e^{-c_{A_{j}}^{A_{k}}(s)}(4 \pi \tau)^{-\frac{n}{2}} \int_{M} e^{-\tilde{f}_{A_{j}}(s)} d V_{h(s)},
\end{gathered}
$$

which implies that $c_{A_{j}}^{A_{k}}(s)=0$ for all $s \in\left[0, A_{j}\right]$ and all $k \geq j$. Therefore, $\tilde{f}_{A_{j}}(s)=\tilde{f}_{A_{k}}(s)$ for all $s \in\left[0, A_{j}\right]$. Define a function $f_{h}(s)$ in the following way. Let $f_{h}(s)=\tilde{f}_{A_{j}}(s)$, for all $s \in\left[0, A_{j}\right]$ and all $A_{j} \rightarrow \infty . f_{h}(s)$ is a well defined function because of the previous discussion. We also have that

$$
R(h(s))_{p q}+\nabla_{p} \nabla_{q} f_{h}(s)-\frac{1}{2 \tau} h(s)_{p q}=0,
$$

holds for all $s \in[0, \infty)$. The definition of $f_{h}(s)$ does not depend on a choice of an increasing sequence $A_{j}$. Namely, if $B_{j}$ were another increasing sequence and if $f_{h^{\prime}}(s)$ were functions defined using the sequences $B_{j}$ and $t_{i}\left(t_{i}\right.$ is the same sequence as above), then at each time, both functions $f_{h}(s)$ and $f_{h^{\prime}}(s)$ would satisfy the same equation (3.23) and the same integral normalization condition. Therefore, $f_{h}(s)=f_{h^{\prime}}(s)$ for all $s \in[0, \infty)$. 


\subsection{Some properties of the limit solitons.}

Let $t_{i}$ be any sequence converging to infinity. Then as we have seen earlier, there exists a subsequence such that $g\left(t_{i}+s\right) \rightarrow h(s)$, where $h(s)$ is a Ricci soliton. Let $\hat{R}(h(t))=\min R(h(t))$. We will first state a theorem that Hamilton proved in his paper [10].

Theorem 3.17 (Hamiton). Under the normalized Ricci flow, whenever $\hat{R} \leq 0$, it is increasing, whereas if ever $\hat{R} \geq 0$ it remains so forever.

We will use the proof of Theorem 3.17 to prove the following lemma.

Lemma 3.18. Under the assumptions of Theorem 3.2, $\hat{R}(h(t)) \geq 0, \forall t$, for the limit metric $h(t)$ of any sequence of metrics $g\left(t_{i}\right)$, where $g(t)$ is a solution of

$$
\frac{d}{d t} g_{j k}=-2 R_{j k}(g(t))+\frac{1}{\tau} g_{j k}(t)
$$

Proof. Assume that there exists $t_{0}$ such that $\hat{R}\left(h\left(t_{0}\right)\right)<0$. Without loss of generality assume that $t_{0}=0$. Since $g\left(t_{i}\right) \rightarrow h(0)$ as $i \rightarrow \infty$, there exists $i_{0}$, so that for all $i \geq i_{0} \hat{R}\left(g\left(t_{i}\right)\right)<0$. The evolution equation for $R$ is

$$
\frac{d}{d t} R=\Delta R+2|\dot{\mathrm{Ric}}|^{2}+\frac{2}{n} R\left(R-\frac{n}{2 \tau}\right) .
$$

This implies

$$
\frac{d}{d t} \hat{R} \geq \frac{2}{n} \hat{R}\left(\hat{R}-\frac{n}{2 \tau}\right) .
$$

If $\hat{R} \leq 0$, then $\hat{R}$ is increasing (since $\frac{d}{d t} \hat{R} \geq 0$ ). If $\hat{R} \geq 0$ at some time it can not go negative at later times. If there existed $t>t_{i_{0}}$ such that $\hat{R}(g(t)) \geq 0$, then $\hat{R} \geq 0$ would remain so forever, for all $s \geq t$ and therefore, we could not have $\hat{R}\left(g\left(t_{i}\right)\right)<0$ for $t_{i}>t$. That contradicts the fact that $\hat{R}\left(g\left(t_{i}\right)\right)<0$ for all $i \geq i_{0}$. Therefore, $\forall t \geq t_{i_{0}}$ we have that $\hat{R}(g(t))<0$.

$$
\frac{d \hat{R}}{d t} \geq \frac{2}{n} \hat{R}\left(\hat{R}-\frac{n}{2 \tau}\right) \geq 0
$$


for all $t$ big enough. That implies $\hat{R}$ is increasing and therefore, there exists $\lim _{t \rightarrow \infty} \hat{R}(g(t))=-C \leq 0$. Moreover, $\hat{R}(h(s))=-C$ for all $s$. Since $\lim _{i \rightarrow \infty} \hat{R}\left(g\left(t_{i}\right)\right)=\hat{R}(h(0))<0, C>0$. We also have that

$$
\frac{d \hat{R}(h(s))}{d s} \geq-\frac{2}{n} \hat{R}(h(s))\left(\frac{n}{2 \tau}-\hat{R}(h(s))\right)=\frac{2}{n} C\left(\frac{n}{2 \tau}+C\right) \geq 0 .
$$

The left-hand side of the above inequality is zero and therefore, we get that $C=-\frac{n}{2 \tau}$ or $C=0$. Since $C>0$, we get a contradiction. Therefore, $R(h(t)) \geq 0$ for all $t$, what we wanted to prove.

Remark 3.19. Let $(M, g)$ be a compact manifold and $g(t)$ be a Ricci flow on $M$. Since

$$
\frac{d}{d t} \mathcal{W}=\int_{M} 2 \tau\left|R_{i j}+\nabla_{i} \nabla_{j} f-\frac{1}{2 \tau} g_{i j}\right|^{2}(4 \pi \tau)^{-\frac{n}{2}} e^{-f} d V
$$

$\mathcal{W}(g, f, \tau)=$ const along the flow, if $g$ is a Ricci soliton satisfying the equation

$$
R_{i j}+\nabla_{i} \nabla_{j} f-\frac{1}{2 \tau} g_{i j}=0 .
$$

Let $t_{i} \rightarrow \infty$ and $s_{i} \rightarrow \infty$ be two sequences such that $g\left(t_{i}+t\right) \rightarrow h(t)$ and $g\left(s_{i}+t\right) \rightarrow h^{\prime}(t)$ where $h(t)$ and $h^{\prime}(t)$ are 2 Ricci solitons on $M$ that have been constructed earlier. We have proved that

$$
\begin{aligned}
& R_{j k}(h)+\nabla_{j} \nabla_{k} f_{h}(t)-\frac{1}{2 \tau} h_{j k}=0, \\
& R_{j k}\left(h^{\prime}\right)+\nabla_{j} \nabla_{k} f_{h^{\prime}}(t)-\frac{1}{2 \tau} h_{j k}^{\prime}=0,
\end{aligned}
$$

where

$$
\begin{aligned}
f_{h}(t) & =\lim _{j \rightarrow \infty} \lim _{i \rightarrow \infty} f_{A_{j}+t_{i}}\left(t_{i}+t\right), \\
f_{h^{\prime}}(t) & =\lim _{j \rightarrow \infty} \lim _{i \rightarrow \infty} f_{B_{j}+s_{i}}\left(s_{i}+t\right),
\end{aligned}
$$

for some increasing sequences $A_{j} \rightarrow \infty$ and $B_{j} \rightarrow \infty$. By Remark 3.19, we know that $\mathcal{W}\left(h(t), f_{h}(t), \tau\right)=C_{1}$ and $W\left(h^{\prime}(t), f_{h^{\prime}}(t), \tau\right)=C_{2}$ are constant along the flows $h(t)$ and $h^{\prime}(t)$ respectively. 
Lemma 3.20. $C_{1}=C_{2}$, i.e. $\mathcal{W}\left(h(t), f_{h}(t), \tau\right)$ is a same constant for all solitons $h(t)$ that arise as limits of sequences of metrics of our original flow $g(t)$ (3.1) on a compact manifold $M$.

Proof.

$$
\begin{aligned}
\mathcal{W}\left(g\left(t_{i}+t\right), f_{t_{i}+A_{j}}\left(t_{i}+t\right), \tau\right)-\mathcal{W} & \left(g\left(s_{i}\right), f_{s_{i}+B_{j}}\left(s_{i}\right), \tau\right) \\
\leq & \mathcal{W}\left(g\left(t_{i}+A_{j}\right), f_{t_{i}+A_{j}}\left(t_{i}+A_{j}\right), \tau\right) \\
& -\mathcal{W}\left(g\left(s_{i}\right), f_{s_{i}}\left(s_{i}\right), \tau\right) \\
= & \mu\left(g\left(t_{i}+A_{j}\right), \tau\right)-\mu\left(g\left(s_{i}\right), \tau\right) \rightarrow 0,(3 .
\end{aligned}
$$

where we have used the fact that $\mathcal{W}(g(t), f(t), \tau)$ increases in $t$ along the flow (3.1) and the fact that $f_{s_{i}}\left(s_{i}\right)=f_{s_{i}}$ is a minimizer for $\mathcal{W}\left(g\left(s_{i}\right), f, \tau\right)$ over all $f$ belonging to a set $\left\{f \mid \int_{M}(4 \pi \tau)^{-\frac{n}{2}} e^{-f} d V_{g\left(s_{i}\right)}\right\}$. Similarly,

$$
\begin{aligned}
\mathcal{W}\left(g\left(t_{i}+t\right), f_{t_{i}+A_{j}}\left(t_{i}+t\right), \tau\right)-\mathcal{W}\left(g\left(s_{i}\right), f_{s_{i}+B_{j}}\left(s_{i}\right), \tau\right) \\
\geq \mathcal{W}\left(g\left(t_{i}+t\right), f_{t_{i}+t}\left(t_{i}+t\right), \tau\right) \\
-\mathcal{W}\left(g\left(s_{i}+B_{j}\right), f_{s_{i}+B_{j}}\left(s_{i}+B_{j}\right), \tau\right) \\
=\mu\left(g\left(t_{i}+t\right), \tau\right) \\
-\mu\left(g\left(s_{i}+B_{j}\right), \tau\right) \rightarrow 0,
\end{aligned}
$$

when $i \rightarrow \infty$. From equations (3.24) and (3.25), letting $i \rightarrow \infty$, we get

$$
\begin{aligned}
& \mathcal{W}\left(h(t), \tilde{f}_{A_{j}}(t), \tau\right)-\mathcal{W}\left(h^{\prime}(0), \tilde{f}_{B_{j}}^{\prime}(0), \tau\right) \leq 0 . \\
& W\left(h(t), \tilde{f}_{A_{j}}(t), \tau\right)-W\left(h^{\prime}(0), \tilde{f}_{B_{j}}^{\prime}(0), \tau\right) \geq 0 .
\end{aligned}
$$

Let $j \rightarrow \infty$ to get

$$
C_{1}=\mathcal{W}\left(h(t), f_{h}(t), \tau\right)=\mathcal{W}\left(h^{\prime}(0), f_{h^{\prime}}(0), \tau\right)=C_{2}
$$


Lemma 3.21. For every Ricci soliton $h(t)$ that arises as a limit of some sequence of metrics of our original flow $g(t)$, the corresponding function $f_{h}(t)$, that we have constructed before, is a minimizer for Perelman's functional $\mathcal{W}$ with respect to a metric $h(t)$.

Proof. We will first prove the following claim.

Claim 3.22. There exists a sequence $t_{i} \rightarrow \infty$ such that $g\left(t_{i}+t\right) \rightarrow h(t)$ as $i \rightarrow \infty$, where $h(t)$ is a Ricci soliton satisfying $R_{j k}(h)+\nabla_{j} \nabla_{k} f_{h}-\frac{1}{2 \tau} h_{j k}=0$ and $f_{h}(t)$ is a minimizer for $\mathcal{W}(h(t), f, \tau)$.

Proof of the Claim. Let $H(t)=(4 \pi \tau)^{-n / 2} \int_{M} 2 \tau\left|R_{i} j+\nabla_{i} \nabla_{j} f_{t}-\frac{1}{2 \tau} g_{i j}\right|^{2} d t$, where $f_{t}$ is a function such that $\mu(g(t), \tau)=W\left(g(t), f_{t}, \tau\right)$. If we flow $f_{t}$ backward by the equation

$$
\frac{d}{d t} f=-\Delta f+|\nabla f|^{2}-R+\frac{n}{2 \tau}
$$

starting at time $t$, for every $t>0$, we get solutions $f_{t}(s)$. Look at $F_{t}(s)=$ $\mathcal{W}\left(g(s), f_{t}(s), \tau\right)$. We know that

$$
\frac{d}{d s} F_{t}(s)=(4 \pi \tau)^{-\frac{n}{2}} \int_{M} 2 \tau\left|R_{j k}+\nabla_{j} \nabla_{k} f_{t}(s)-\frac{1}{2 \tau} g(s)_{j k}\right|^{2} d V_{g(s)} .
$$

$F_{t}(s)$ is a continuous function in $s \in[0, t]$ and $\lim _{s \rightarrow t} \frac{d}{d s} F_{t}(s)=H(t)$. Therefore, there exists a left derivative of $F_{t}(s)$ at point $t$ and $\left(F_{t}\right)_{-}^{\prime}(t)=H(t)$ for every $t>0$. Moreover, $g(t)$ and all the derivatives of $f_{t}$ up to the second order are Lipshitz functions in $t$ (this follows from the estimates in the previous subsections) and therefore

$$
\mu(t):=\mu(g(t), \tau)=\inf _{\left\{f \mid \int_{M}(4 \pi \tau)^{-\frac{n}{2}} e^{-f}=1\right\}} \mathcal{W}(g(t), f, \tau)
$$

is a Lipshitz function in $t$ as well, i.e. $k(t)=F_{t}(t)=\mathcal{W}\left(g(t), f_{t}, \tau\right)$ is a Lipshitz function in $t$. This tells that $k(t)$ is differentiable in $t$, almost 
everywhere. Our discussion then implies that $k^{\prime}(t)=H(t)$ in a sense of distributions.

$$
\begin{aligned}
\int_{\delta}^{\infty} H(t) d t & =\lim _{K \rightarrow \infty} \int_{\delta}^{K} k^{\prime}(t) d t \\
& =\lim _{K \rightarrow \infty} W\left(g(K), f_{K}, \tau\right)-W\left(g(\delta), f_{\delta}, \tau\right) \\
& =\lim _{K \rightarrow \infty}(\mu(g(K), \tau)-\mu(g(\delta), \tau) \leq C,
\end{aligned}
$$

where $\delta>0$ and $C$ is some uniform constant. We have that $\int_{\delta}^{\infty} H(t) \leq C$. This implies that there exists a sequence $t_{i} \rightarrow \infty$ such that $H\left(t_{i}\right) \rightarrow 0$ as $i \rightarrow \infty$, i.e.

$$
\lim _{i \rightarrow \infty}\left(R_{j k}+\nabla_{j} \nabla_{k} f_{t_{i}}-\frac{1}{2 \tau} g_{j k}\right)\left(t_{i}\right)=0 .
$$

By what we have proved before, after extracting a subsequence, we can assume that $g\left(t_{i}\right) \rightarrow h(0)$ smoothly and $f_{t_{i}} \rightarrow \tilde{f}$ in $C^{2, \alpha}$ norm, where by Theorem $3.8 \tilde{f}$ is a minimizer for $\mathcal{W}$ with respect to metric $h(0)$. Therefore,

$$
R_{j k}(h(0))+\nabla_{j} \nabla_{k} \tilde{f}-\frac{1}{2 \tau} h_{j k}(0)=0 .
$$

On the other hand, $g\left(t_{i}+t\right) \rightarrow h(t)$ as $i \rightarrow \infty$ where $h(t)$ is a Ricci soliton and

$$
R_{j k}(h(t))+\nabla_{j} \nabla_{k} f_{h}(t)-\frac{1}{2 \tau} h_{j k}(t)=0,
$$

where $f_{h}(t)=\lim _{j \rightarrow \infty} \lim _{i \rightarrow \infty} f_{t_{i}+A_{j}}\left(t_{i}+t\right)$, for some sequence $A_{j} \rightarrow \infty$. From equations (3.27) and (3.28), we have that $\Delta\left(f_{h}(0)-\tilde{f}\right)=0$, i.e. $f_{h}(0)=\tilde{f}+C$ for some constant $C$. We know that $\int_{M}(4 \pi \tau)^{-\frac{n}{2}} e^{-\tilde{f}} d V_{h(0)}=1$, since $\tilde{f}$ is a minimizer. From the construction of $f_{h}(t)$ it follows that $\int_{M}(4 \pi \tau)^{-\frac{n}{2}} e^{-f_{h}(0)} d V_{h(0)}=1$ and therefore, $\tilde{f}=f_{h}(0)$. Since there exists a finite limit, $\lim _{t \rightarrow \infty} \mu(g(t), \tau)$, we have that $\mu(h(0), \tau)=\mu(h(t), \tau)$ for all $t$. This implies that

$$
\begin{aligned}
\mu(h(t), \tau) & =\mu(h(0), \tau)=\mathcal{W}(h(0), \tilde{f}, \tau) \\
& =\mathcal{W}\left(h(0), f_{h}(0), \tau\right)=\mathcal{W}\left(h(t), f_{h}(t), \tau\right),
\end{aligned}
$$


where we have used the fact that $\mathcal{W}$ is constant along a soliton. This means that $f_{h}(t)$ is a minimizer for $\mathcal{W}$ with respect to a metric $h(t)$, for every $t \geq 0$.

To continue the proof of Lemma 3.21 take any sequence $s_{i} \rightarrow \infty$. By a sequential convergence of our original flow $g(t)$ to Ricci solitons, after extracting a subsequence, we may assume that $g\left(s_{i}+t\right) \rightarrow h^{\prime}(t)$ as $i \rightarrow \infty$ where $h^{\prime}(t)$ is a Ricci soliton. Take a soliton $h(t)$ with the properties as in Claim 3.22. From the convergence of $\mu(g(t), \tau)$, we know that $\mu\left(h^{\prime}(t), \tau\right)=$ $\mu(h(s), \tau)$ for all $t$ and all $s$.

$$
\mu\left(h^{\prime}(t), \tau\right)=\mu(h(s), \tau)=\mathcal{W}\left(h(s), f_{h}(s), \tau\right) .
$$

By Lemma 3.20, we have that $\mathcal{W}\left(h(s), f_{h}(s), \tau\right)=\mathcal{W}\left(h^{\prime}(t), f_{h^{\prime}}(t), \tau\right)$ for all $s$ and $t$. Combining this with (3.29) gives that $\mu\left(h^{\prime}(t), \tau\right)=\mathcal{W}\left(h^{\prime}(t), f_{h^{\prime}}(t), \tau\right)$, i.e. $f_{h^{\prime}}(t)$ is a minimizer for $h^{\prime}(t)$ for every $t$.

One useful property of the sequential soliton limits of our flow (3.1) is that all limit solitons are the solutions of the normalized flow equation

$$
\frac{d}{d t} h_{i j}=-2 R_{i j}+\frac{2}{n} r(h(t)) h_{i j}
$$

where $r(h(t))=\frac{1}{\operatorname{Vol}_{h(t)} M} \int_{M} R(h(t)) d V_{h(t)}$. In the case of any of our soliton limits, we have that $R(h(t))+\Delta f_{h}(t)-\frac{n}{2 \tau}=0$ and therefore, $r=r(h(t))=\frac{n}{2 \tau}$ for all $t \geq 0$.

Remark 3.23. Let $t_{i} \rightarrow \infty$ and $g\left(t_{i}+t\right) \rightarrow h(t)$, where $h(t)$ is an Einstein metric with an Einstein constant $\frac{1}{2 \tau}$. If $\operatorname{Vol}_{h^{\prime}}(M)=\operatorname{Vol}_{h}(M)$, for any other limit soliton $h^{\prime}$, then $h^{\prime}$ is an Einstein metric with the same Einstein constant $\frac{1}{2 \tau}$.

Proof. The fact that $h$ is Einstein metric implies that $\nabla_{i} \nabla_{j} f_{h}=-2 R_{i j}+$ $\frac{1}{\tau} h_{i j}=0$, that is $\Delta f_{h}=0$. Since $M$ is compact, $f_{h}=C$ such that $(4 \pi \tau)^{-n / 2} e^{-C} \operatorname{Vol}_{h}(M)=1$. An easy computation shows that $\mu(h, \tau)=$ 
$\mathcal{W}(h, C, \tau)=C-\frac{n}{2}$, and therefore, $\mu\left(h^{\prime}, \tau\right)=\mu(h, \tau)=C-\frac{n}{2}$. Then, $(4 \pi \tau)^{-n / 2} e^{-C} \operatorname{Vol}_{h^{\prime}}(M)=1$, implies that $f=C$ is a minimizer for $\mathcal{W}$ with respect to $h^{\prime}$ as well. This yields

$$
\tau\left(2 \Delta f-|\nabla f|^{2}+R\left(h^{\prime}\right)\right)+f-n=C-\frac{n}{2},
$$

that is

$$
R\left(h^{\prime}\right)=\frac{n}{2 \tau}
$$

From

$$
\Delta f_{h^{\prime}}=\frac{n}{2 \tau}-R\left(h^{\prime}\right)=0,
$$

we get that $f_{h^{\prime}}=C$ and therefore

$$
R_{i j}\left(h^{\prime}\right)+\nabla_{i} \nabla_{j} f_{h^{\prime}}-\frac{1}{2 \tau} h_{i j}^{\prime}=0,
$$

yields $R_{i j}\left(h^{\prime}\right)=\frac{1}{2 \tau} h_{i j}^{\prime}$.

In the discussion that follows, we will use Moser's weak maximum principle. We will state it below, for a reader's convenience.

Lemma 3.24 (Moser's weak maximum principle). Let $g=g(t), 0 \leq$ $t<T$, be a smooth family of metrics, $b$ a non-negative constant and $f a$ non-negative function on $M \times[0, T)$ which satisfies the partial differential inequality

$$
\frac{d f}{d t} \leq \Delta f+b f
$$

on $M \times[0, T]$, where $\Delta$ refers to a Laplacian at time $t$. Then for any $x \in M$, $t \in[0, T)$,

$$
|f(x, t)| \leq c \frac{1}{\sqrt{V}} e^{c H d} \max \left(1, d^{\frac{n}{2}}\right)\left(b+l+\frac{1}{t}\right)^{\frac{1+n / 2}{2}} e^{c b t}|| f_{0} \|_{L^{2}},
$$

where $c$ is a positive constant depending only on $n$ and $d=$ $\max _{0 \leq t \leq T} \operatorname{diam}(M, g(t)), H=\max _{0 \leq t \leq T} \sqrt{\|\operatorname{Ric}\|_{C^{0}}}, f_{0}=f(\cdot, 0), \quad V=$ $\min _{0 \leq t \leq T} \operatorname{Vol}_{g(t)}(M)$.

The following remark will give us a condition that will imply obtaining the Einstein metrics in the limit. 
Remark 3.25. If $g(t)$ is a solution to $\left(g_{i j}\right)_{t}=-2 R_{i j}+\frac{1}{\tau} g_{i j}$, for $t \in[0, \infty)$ such that

1. A curvature operator and a diameter are uniformly bounded along the flow.

2. $0 \leq R(x, t) \leq \frac{n}{2 \tau}$ for all $x \in M$ and all $t \in[0, \infty)$.

Then all the solitons that arise as limits of the subsequences of our flow $g(t)$ are Einstein metrics with scalar curvatures $R=\frac{n}{2 \tau}$ and $T_{i j}(t)$ converge to zero, uniformly on $M$ as $t \rightarrow \infty$. $T_{i j}=R_{i j}-\frac{R}{n} g_{i j}$ is a traceless part of the Ricci curvature.

Proof of the Remark. Notice that now we do not make an assumption that one of the metrics that we get in a limit is an Einstein metric. Look at the evolution equation for $r(t)=\frac{1}{\operatorname{Vol}_{t}(M)} \int_{M} R d V_{t}$,

$$
\frac{d}{d t} r(t)=\frac{1}{\operatorname{Vol}_{t}(M)}\left(2 \int_{M}|T|^{2}+\left(1-\frac{2}{n}\right) \int_{M} R\left(\frac{n}{2 \tau}-R\right)+r\left(r-\frac{n}{2 \tau}\right) .\right.
$$

$R \leq \frac{n}{2 \tau}$ implies $r(t) \leq \frac{n}{2 \tau}$ and therefore

$$
\frac{d}{d t} r(t) \geq \frac{2}{\operatorname{Vol}_{t}(M)} \int_{M}|T|^{2}+r\left(r-\frac{n}{2 \tau}\right) .
$$

We have proved that in the case of flow $g(t)$, a volume non-collapsing condition holds for all times $t \geq 0 . \frac{d}{d t} \ln \left(\operatorname{Vol}_{t}(M)\right)=\frac{n}{2 \tau}-r$ and $C_{1} \leq \operatorname{Vol}_{t}(M) \leq$ $C_{2}$ give that $\int_{0}^{\infty}\left(\frac{n}{2 \tau}-r(t)\right) d t<\infty$. We can integrate the inequality (3.30) in $t \in[0, \infty)$. This, together with the uniform estimates on $\operatorname{Vol}_{t}(M)$ and $r(t)$ give that

$$
\int_{0}^{\infty} \int_{M}|T|^{2} d V_{t} \leq C
$$

Following the calculations in Hamilton's paper [6], Rugang computed the evolution equation for $T$ under a normalized Ricci flow ([18]). In the case of flow (3.1), we have

$$
\frac{d}{d t}|T|^{2}=\Delta|T|^{2}-2|\nabla T|^{2}+4 \operatorname{Rm}(T) \cdot T+\frac{4}{n}\left(R-\frac{n}{2 \tau}\right)|T|^{2} .
$$


Since the curvature operators of $g(t)$ are uniformly bounded, we derive from equation (3.32) that

$$
\frac{d}{d t}|T| \leq \Delta|T|+C|T|
$$

Applying Lemma 3.24 to this differential inequality and intervals $[t-1, t+1]$ where $t>1$, we derive

$$
|T|^{2}(x, t) \leq\|T\|^{2}(t)_{C^{0}(M)} \leq C\left(\int_{M_{t-1}}|T|^{2}\right)
$$

where $M_{t}=(M, g(t))$. Integrate this inequality in $t \in[k, k+1]$, for all $k \geq k_{0}$ and sum up all the inequalities that we get this way. We get

$$
\begin{gathered}
\int_{k_{0}}^{\infty}\|T\|^{2} d t \leq C \sum_{k \geq k_{0}} \int_{k}^{k+1}\left(\int_{M_{t-1}}|T|^{2}\right) d t \\
\int_{k_{0}}^{\infty}\|T\|^{2} d t \leq C \int_{k_{0}}^{\infty} \int_{M}|T|^{2} d V_{t-1} d t
\end{gathered}
$$

where $d V_{t-1}$ is a volume form for metric $g(t-1) . \quad \int_{M}|T|^{2} d V_{t-1} \leq$ $C \int_{M}|T|^{2} d V_{t}$, because $\frac{d}{d t} \ln \operatorname{Vol}_{t}=\frac{n}{2 \tau}-R$ and the curvatures of $g(t)$ are uniformly bounded. The right-hand side of inequality (3.33) is bounded by a uniform constant, because of the estimate (3.31). Therefore, $\int_{k_{0}}^{\infty}\|T\|^{2} d V_{t}$ $\leq C$.

If there exists $\left(p, t_{0}\right)$ such that $|T|^{2}\left(p, t_{0}\right)>\epsilon$, then there is a small neighbourhood of $\left(p, t_{0}\right)$ in $M \times[0, \infty)$, say $U_{\delta}\left(p, t_{0}\right)=B_{p}\left(\delta, t_{0}\right) \times\left[t_{0}-\delta, t_{0}+\delta\right]$ such that $|T|^{2}(x, t) \geq \frac{\epsilon}{2}$ for all $(x, t) \in U_{\delta}\left(p, t_{0}\right)$. This follows from the fact that in the case of a Ricci flow, a bound $|\mathrm{Rm}| \leq C$ implies $\left|D^{k} D_{t}^{l} \mathrm{Rm}\right| \leq$ $C(k, l)$. Costant $\delta$ does not depend on a point $\left(p, t_{0}\right) \in M \times[0, \infty)$, since all our bounds and estimates are uniform.

If there existed $\epsilon>0$ and a sequence of points $\left(p_{i}, t_{i}\right) \in M \times[0, \infty)$, with $t_{i} \rightarrow \infty$ such that $\left|T\left(p_{i}, t_{i}\right)\right| \geq \epsilon$, then we would have that $\|T\|_{C^{0}} \geq \frac{\epsilon}{2}$ for all $t \in\left[t_{i}-\delta, t_{i}+\delta\right]$ and for all $i$. This would imply $C \geq \int_{0}^{\infty}\|T\|^{2} d V_{t} \geq$ $\sum_{i=0}^{\infty} \epsilon \delta=\infty$. This is impossible. Therefore, $\|T\|_{C^{0}\left(M_{t}\right)} \rightarrow 0$ as $t \rightarrow \infty$. 
$\frac{d}{d t} \ln \left(\mathrm{Vol}_{t}\right)=\frac{n}{2 \tau}-R \geq 0$ for all $t$ imply that there exists a finite $\lim _{t \rightarrow \infty} \operatorname{Vol}_{t}$ for every $x \in M$ (otherwise, we can argue as in the previous paragraph). If we integrate this equation in $t \in[0, \infty)$, we will get that $\int_{0}^{\infty}\left(\frac{n}{2 \tau}-R\right) d t<\infty$. As in the case for a traceless part of the Ricci curvature $T$, we can conclude that $\lim _{t \rightarrow \infty} R=\frac{n}{2 \tau}$ uniformly on $M$.

We can conclude that under the assumptions given at the beginning of this remark, for every sequence $t_{i} \rightarrow \infty$, we can find a subsequence such that $g\left(t_{i}+t\right) \rightarrow h(t)$, where $h(t)$ is an Einstein soliton with scalar curvature $\frac{n}{2 \tau}$. We also know that $R_{i j}-\frac{1}{2 \tau} g_{i j} \rightarrow 0$ as $t \rightarrow \infty$, uniformly on $M$ and that there exists $\lim _{t \rightarrow \infty} \mathrm{Vol}_{t}$.

To conclude, we have proved a sequential convergence of a solution of a $\tau$-flow towards solitons (generalizations of Einstein metrics), under uniform curvature and diameter assumptions. We still do not know whether we get a unique soliton (up to diffeomorphisms) in the limit or not. All observations in this subsection are in favor of the uniqueness of a soliton in the limit.

\section{Uniqueness of a limit soliton.}

In this section, we will assume that one of the limit solitons is integrable, in order to prove the uniqueness of a soliton in the limit, up to a diffeomorphism. We will first construct a gauge in which a $\tau$-flow becomes a strictly parabolic flow. Similar ideas to those in [3] will help us finish the proof of Theorem 1.1.

\subsection{The construction of a gauge.}

To construct the right gauge, assume for simplicity that we are in a situation when $g(t) \rightarrow h$ as $t \rightarrow \infty$, where $h$ is an Einstein metric, with the Einstein

constant $\frac{1}{2 \tau}$. We will see how we construct a gauge so that our modified Ricci flow equation becomes strictly parabolic on time intervals of an arbitrary length, if we go sufficiently far in time direction. This construction applies to our more general case, just with minor modifications and only for simplicity reasons, we have decided to consider a case of an Einstein metric in a limit. The main purpose of this section is to prove the following Proposition that will be reformulated in the next section for our more general setting. 
Proposition 4.1. Let $A>0$ be an arbitrary real number, $k$ an integer and $0<\alpha<1$. There exists $\epsilon_{0}(A, k)$ such that for every $\epsilon<\epsilon_{0}$, there exists $s_{0}=s_{0}(A, k, h, \epsilon)$, such that for all $t_{0} \geq s_{0}$ the equation

$$
\begin{aligned}
\frac{d}{d t} \phi & =\Delta_{g(t), h} \phi, \\
\phi\left(t_{0}\right) & =\phi_{t_{0}},
\end{aligned}
$$

has a solution $\phi(t)$, so that it is a diffeomorphism, $|\phi(t)-\mathrm{Id}|_{k, \alpha, h}<\epsilon$ and $\left|\phi^{*} g(t)-h\right|_{k, \alpha}<\epsilon$, for every $t \in\left[t_{0}, t_{0}+A\right] . \phi_{t_{0}}$ is chosen to be a diffeomorphism so that $\delta_{\phi^{*}\left(t_{0}\right) h}\left(g\left(t_{0}\right)\right)=0$.

Definition 4.2. Let $\phi: M \rightarrow M$ be a smooth function. Define $e(\phi-\mathrm{Id})=$ $g^{i j} h_{k l}\left(\phi_{i}^{k}-\operatorname{Id}_{i}^{k}\right)\left(\phi_{j}^{l}-\operatorname{Id}_{j}^{l}\right)$. Define $E(\phi-\mathrm{Id})=\int_{M} e(\phi-\mathrm{Id})$ and $F_{l}=\phi^{l}-\mathrm{Id}^{l}$.

Throughout the proof of Proposition 4.1, we will have a tendency to use the same symbol for different uniform constants.

Proof of Proposition 4.1. Fix $A>0$. Let $\epsilon>0$ be very small (we will see later how small we want to take it). We know that for $s_{0}$ sufficiently big, we can make $|g(t)-h|$ as small as we want, and therefore, we have that $\delta_{\phi\left(t_{0}\right)^{*} h} g\left(t_{0}\right)=0$ implies that $\left|\phi\left(t_{0}\right)-\mathrm{Id}\right|_{k+2, \alpha, h}<\epsilon / 1000$ on $M$ (see [3] for more details). Choose some $t_{0} \geq s_{0}$. We can make $\left|F\left(t_{0}\right)\right|_{N, \alpha, h}$, for say $N>>k$ as small as we want by choosing $s_{0}$ sufficiently big. Since $g(t) \rightarrow h$ as $t \rightarrow \infty$, the coefficients and the initial data of harmonic map flow (4.1) are uniformly bounded and uniformly close to each other for $t_{0}$ big enough. This implies that there exists a uniform constant $\delta_{1}>0$ so that a solution to (4.1) exists on $t \in\left[t_{0}, t_{0}+\delta_{1}\right)$, for all $t_{0} \geq s_{0}$. For the same reasons there exists some $\delta>0$ such that $|F(t)|_{W^{2, N}, g(t)}<\epsilon$, for $t \in\left[t_{0}, t_{0}+\delta\right)$. We can assume that we have chosen $N$ big enough so that as a consequence of Sobolev embedding theorems, we have that $|F|_{k, \alpha, g(t)}<\tilde{\epsilon}(\tilde{\epsilon}$ differs from $\epsilon$ by a Sobolev embedding constant) for all $t \in\left[t_{0}, t_{0}+\delta\right)$ and all $t_{0} \geq s_{0}$. We want to show that the estimate $|F(t)|_{W^{2, N}, g(t)}<\epsilon$ holds past time $t_{0}+\delta$, until $\delta<A$. Then $|F|_{k, \alpha, g(t)}<\tilde{\epsilon}$ continues to hold past time $t_{0}+\delta$, until 
$\delta<A$. This actually gives a uniform upper bound on the energy densities on whole manifold $M$. To see this, notice that a bound $|F|_{k, \alpha}<\tilde{\epsilon}$ implies that $e(\phi-\mathrm{Id}) \leq C \tilde{\epsilon}$. Since

$$
e(\phi-\mathrm{Id})=e(\phi)+e(\mathrm{Id})-2 g^{i j} h_{k l} \mathrm{Id}_{i}^{k} \phi_{j}^{l},
$$

by the Schwartz inequality for quadratic forms and the interpolation inequality, we get that

$$
\begin{aligned}
e(\phi) & \leq C \tilde{\epsilon}+C+2\left(g^{i j} h^{k l} \phi_{i}^{k} \phi_{j}^{l}\right)^{1 / 2}\left(g^{i j} h^{k l} \operatorname{Id}_{i}^{k} \operatorname{Id}_{j}^{l}\right)^{1 / 2} \\
& \leq C \tilde{\epsilon}+C+\eta e(\phi)
\end{aligned}
$$

for some $\eta<1$, which implies that $e(\phi) \leq \tilde{C}$. By the results proved by Eells and Sampson in [5] there exists $\bar{\delta}$, depending on $(M, h)$ and the uniform bound on the energy densities $\tilde{C}$, so that for every $s \in\left[t_{0}, t_{0}+\delta\right)$ a solution to a harmonic map flow (4.1) can be extended to $[s, s+\bar{\delta}]$. If $t_{0}+\delta+\bar{\delta}<t_{0}+A$, we can repeat the procedure above for a solution $\phi(t)$, on time interval $\left[t_{0}, t_{0}+\delta+\bar{\delta}\right)$ to get that the energy density estimates with the same constant $\tilde{C}$ hold past time $t_{0}+\delta+\bar{\delta}$. Since all our estimates depend only on $A$ and the uniform bounds on geometries $g(t)$, we can iterate the argument till we reach time $t_{0}+A$, for every $t_{0} \geq s_{0}$. As a result, we will get $\phi(t)$, a solution to (4.1), such that $|\phi(t)-\mathrm{Id}|_{k, \alpha}<\tilde{\epsilon}$ for all $t \in\left[t_{0}, t_{0}+A\right]$.

We know that $\left(\Delta_{g(t), h} \mathrm{Id}\right)^{\gamma}=g^{\alpha \beta}\left(\Gamma(h)_{\alpha \beta}^{\gamma}-\Gamma(g)_{\alpha \beta}^{\gamma}\right)$ and that $\frac{d}{d t} \operatorname{Id}=0$. Therefore, we have

$$
\frac{d}{d t}\left(\phi^{k}-\mathrm{Id}^{k}\right)=\Delta_{g(t), h}\left(\phi^{k}-\mathrm{Id}^{k}\right)+g^{i j}\left(\Gamma_{i j}^{k}(h)-\Gamma_{i j}^{k}(g)\right),
$$

where we can choose $s_{0}$ so big, that the last term is arbitrarily small (since $g(t) \rightarrow h)$. We will see later how small we want to make it, for now we can say it is less than some $\epsilon_{1}>0$.

Before we start establishing the estimates on $F=\phi-\mathrm{Id}$, we will occupy ourselves with the problem of replacing equation (4.2) which in terms of local coordinates on $M$ is a local system of equations, by some much more global 
system. Passing to a global system of equations will make establishing the estimates on $F$ much easier. We will follow a discussion in [5].

Since $M$ is compact, there exists an embedding $\omega: M \rightarrow R^{q}$ and due to Eells and Sampson ([5]) it is always possible to construct a smooth Riemannian metric $g^{\prime \prime}=\left(g_{a b}^{\prime \prime}\right)_{1 \leq a, b \leq q}$ on a tubular neighborhood $N$ of $M$ in $R^{q}$, such that $N$ is Riemannian fibered. They actually meant that if $\pi: N \rightarrow M$ is a projection map, it suffices to construct an appropriate smooth inner product in each space $R^{q}(p)$ for all $p \in M$, for which we can translate that tangent space to any point $m \in N$ along the straight line segment (that is contained in $N$ ) from $p=\pi(m)$ to $m$. Following the arguments of Section 7 in [5], we find that the evolution equation (4.2), given in local coordinates is satisfied by $\phi-$ Id if and only if $W-\tilde{W}$, where $W=\omega \circ \phi$ and $\tilde{W}=\omega \circ \operatorname{Id}$ satisfies

$$
\begin{aligned}
\frac{d}{d t}\left(W^{c}-\tilde{W}^{c}\right)= & \Delta\left(W^{c}-\tilde{W}^{c}\right)+\pi_{a b}^{c}\left(W_{i}^{a}-\tilde{W}_{i}^{a}\right)\left(W_{j}^{b}-\tilde{W}_{j}^{b}\right) g^{i j} \\
& +\frac{\partial \omega^{c}}{\partial y^{k}} g^{i j}\left(\Gamma_{i j}^{k}(h)-\Gamma_{i j}^{k}(g)\right),
\end{aligned}
$$

where $\left(y_{1}, \ldots, y_{n}\right)$ are the local coordinates on $M$. Moreover, since $M$ is compact, the projection $\pi$ satisfies (see [5])

$$
\left|\pi_{a b}^{c}\right|_{k+1, \alpha} \leq C
$$

on $M$ and there are constants $A_{1}$ and $A_{2}$ so that

$$
A_{1} d s_{0}^{2} \leq d s^{2} \leq A_{2} d s_{0}^{2}
$$

where $d s_{0}^{2}$ denotes the line element induced on $M$ by the usual metric on $R^{q}$. These estimates immediately imply that

$$
\left|\frac{\partial^{k} \pi_{a b}^{c}}{\partial y^{k}} W_{i}^{a} W_{j}^{b} g^{i j}\right| \leq C(k) e(\phi),
$$

where also $e(\phi)=g_{a b}^{\prime \prime} W_{i}^{a} W_{j}^{b} g^{i j}, e(\phi-\mathrm{Id})=g_{a b}^{\prime \prime}(W-\tilde{W}) g^{i j}$. Moreover, if $\tilde{F}^{c}=W^{c}-\tilde{W}^{c}$, then $\left|\frac{\partial^{k} \pi_{a b}^{c}}{\partial y^{k}} \tilde{F}_{i}^{a} \tilde{F}_{j}^{b} g^{i j}\right| \leq C e(\phi-\mathrm{Id})$. 
The evolution equation for $e(\phi-\mathrm{Id}$ ) (see for details [5] and [9]) is

$$
\begin{aligned}
\frac{d}{d t} e(\phi-\mathrm{Id})= & \Delta e(\phi-\mathrm{Id})-2\left|D^{2}(\phi-\mathrm{Id})\right|^{2} \\
+ & 2 \operatorname{Rm}(D(\phi-\mathrm{Id}), D(\phi-\mathrm{Id}), D(\phi-\mathrm{Id}), D(\phi-\mathrm{Id})) \\
- & \frac{1}{\tau} e(\phi-\mathrm{Id})+g^{i j} h_{k l}\left(\phi_{j}^{k}-\mathrm{Id}_{j}^{k}\right) \\
& {\left[g^{p q}\left(\Gamma_{p q}^{l}(h)-\Gamma_{p q}^{l}(g)\right)\right]_{i} }
\end{aligned}
$$

where $\operatorname{Rm}(D(\phi-\mathrm{Id}), D(\phi-\mathrm{Id}), D(\phi-\mathrm{Id}), D(\phi-\mathrm{Id}))=g^{i k} g^{j l} R_{p q m n} D_{i}\left(\phi^{p}-\right.$ $\left.\operatorname{Id}^{p}\right) D_{j}\left(\phi^{q}-\operatorname{Id}_{q}\right) D_{k}\left(\phi^{m}-\operatorname{Id}^{m}\right) D_{l}\left(\phi^{n}-\operatorname{Id}^{n}\right)$ and $\left|D^{2}(\phi-\mathrm{Id})\right|^{2}=$ $g^{i k} g^{j l} h_{p q} D_{i j}^{2}\left(\phi^{p}-\mathrm{Id}^{p}\right) D_{k l}^{2}\left(\phi^{q}-\mathrm{Id}^{q}\right)$. Applying the Schwarz inequality for quadratic forms and using the fact that $2 \sqrt{\tau}\left(g^{p q}\left(\Gamma_{p q}^{l}(h)-\Gamma_{p q}^{l}(g)\right)\right)_{i}$ can be made arbitrarily small by choosing $s_{0}$ sufficiently big (e.g. smaller than $\left.\frac{2 \epsilon}{1000}\right)$, the last term in inequality (4.4) can be estimated as

$$
g^{i j} h_{k l}\left(\phi_{j}^{k}-\operatorname{Id}_{j}^{k}\right)\left[g^{p q}\left(\Gamma_{p q}^{l}(h)-\Gamma_{p q}^{l}(g)\right)\right]_{i} \leq \frac{e(\phi-\mathrm{Id})^{\frac{1}{2}}}{2 \sqrt{\tau}}(2 \epsilon) / 1000
$$

Factor of 1000 (that we can increase if necessary) is chosen so that after multiplying $\frac{\epsilon}{1000}$ by at most a polynomial expression in $A$ (which will become more apparent later in the proof of Proposition 4.1) can be made again much smaller than $\epsilon$. Therefore, for $t \in\left[t_{0}, t_{0}+\delta\right)$ we have that

Claim 4.3. There exists $C$, small $\epsilon$ and sufficiently big $s_{0}$ such that for all $t_{0} \geq s_{0}$

$$
\begin{aligned}
& \text { 1. } e(\phi-\mathrm{Id})<\epsilon_{1} \text {, } \\
& \text { 2. } E(\phi-\mathrm{Id})(s)<\epsilon_{1} \text {, }
\end{aligned}
$$

for all $s$ belonging to a time interval starting at $t_{0}$ at which $\phi$ exists, where $\epsilon_{1}$ is a constant that can be made much smaller than $\epsilon$. 
Proof. By using the interpolation inequality in (4.4), we get

$$
\begin{aligned}
\frac{d}{d t} e(\phi-\mathrm{Id}) & \leq \Delta e(\phi-\mathrm{Id})+C \epsilon^{4}-\frac{1}{\tau} e(\phi-\mathrm{Id})+\frac{1}{2 \tau} e(\phi-\mathrm{Id})+C \frac{\epsilon^{2}}{1000^{2}} \\
& \leq \Delta e(\phi-\mathrm{Id})-\frac{1}{2 \tau} e(\phi-\mathrm{Id})+\frac{\epsilon}{1000},
\end{aligned}
$$

since we can start with $\epsilon$ as small as we want, in particular, we may choose $\epsilon$ so that $C \epsilon^{4}+C \frac{\epsilon^{2}}{1000^{2}}<\frac{\epsilon}{1000}$ and increase $s_{0}$ if necessary.

Let $f(t)=\max _{M} e(\phi-\mathrm{Id})(t)$. Then

$$
\begin{gathered}
\frac{d}{d t} f \leq-\frac{1}{2 \tau} f+\frac{\epsilon}{1000}, \\
\frac{d}{d t} f \leq-\frac{1}{2 \tau}\left(f-\frac{\tau \epsilon}{500}\right) .
\end{gathered}
$$

If we choose $s_{0}$ big enough, we may assume that $f\left(t_{0}\right)<\frac{\tau \epsilon}{500} \operatorname{Vol}_{h}(M)$. If $\left.f(t) \geq \frac{\tau \epsilon}{500} \operatorname{Vol}_{h}(M)\right)$ for some $t>t_{0}$, then $f(t)$ is non-increasing (because $\frac{d}{d t} f(t) \leq 0$ and since it starts as $\left.f \leq \frac{\tau \epsilon}{500} \operatorname{Vol}_{h}(M)\right)$ ), it will remain so forever while $\phi$ exists. Denote by $\epsilon_{1}=\frac{\tau \epsilon}{500} \max _{t} \operatorname{Vol}_{g(t)}(M)$.

$$
E(\phi-\mathrm{Id})(s)=\int_{U} e(\phi-\mathrm{Id})(s) d V_{g(s)}<\epsilon_{1}
$$

By Claim 4.3, $e(W-\tilde{W})$ can be made much smaller than $\epsilon$ whenever $\phi$ is defined (if $t_{0}$ is big enough and $\epsilon$ is small enough). The conditions $|F|_{W^{2, N}}<\epsilon$ and $|F|_{k, \alpha}<\tilde{\epsilon}$ actually mean that for $\tilde{F}$, we make an assumption that $|\tilde{F}|_{W^{2, N}}<\epsilon$ and $|\tilde{F}|_{k, \alpha}<\tilde{\epsilon}$, for $t \in\left[t_{0}, t_{0}+\delta\right.$ ) (these $\epsilon$ and $\tilde{\epsilon}$ can be slightly different from those for $F$ ). In order to finish the proof of Proposition 4.1 , it is enough to show that $|\tilde{F}|_{k, \alpha}<\tilde{\epsilon}$ continues to hold past time $t_{0}+\delta$, for $t_{0}$ big enough. From now on, we will consider a globally defined evolution equation

$$
\frac{d}{d t}\left(\tilde{F}^{c}\right)=\Delta \tilde{F}^{c}+\pi_{a b}^{c} \tilde{F}_{i}^{a} \tilde{F}_{j}^{b} g^{i j}+\frac{\partial \omega^{c}}{\partial y^{k}} g^{i j}\left(\Gamma_{i j}^{k}(h)-\Gamma_{i j}^{k}(g)\right),
$$


Step 4.4. $\int_{M}\left|\tilde{F}^{c}\right|^{2} d V_{g(t)}$ and $\int_{t_{0}}^{t_{0}+\delta} \int_{M}\left|\nabla \tilde{F}^{c}\right|^{2} d V_{g(t)}$ can be made much smaller than $\epsilon$, for all $t \in\left[t_{0}, t_{0}+\delta\right)$ and for all $t_{0}$ big enough.

Multiply the equation (4.6) by $\tilde{F}^{c}$ and integrate it over $M$ against the metric $g(t)$.

$$
\begin{aligned}
\frac{1}{2} \frac{d}{d t} \int\left(\tilde{F}^{c}\right)^{2} d V_{g(t)}< & \int_{M}\left(\tilde{F}^{c}\right)^{2}\left(\frac{n}{2 \tau}-R\right) d V_{g(t)}-\int_{M}\left|\nabla \tilde{F}^{c}\right|^{2} d_{g(t)} \\
& +\epsilon_{1} \int_{M}\left|\tilde{F}^{c}\right| d V_{g(t)} \\
& +C\left(\int_{M} e(\tilde{F})^{2} d V_{g(t)}\right)^{1 / 2}\left(\int_{M}\left(\tilde{F}^{c}\right)^{2} d V_{g(t)}\right)^{1 / 2} \\
\leq & \epsilon_{1} \epsilon-\int_{M}\left|\nabla \tilde{F}^{c}\right|^{2}+\epsilon_{1}\left[\int_{M}\left|\tilde{F}^{c}\right| d V_{g(t)}\right] \\
& +C \epsilon \epsilon_{1} \int_{M}\left(\tilde{F}^{c}\right)^{2} d V_{g(t)}
\end{aligned}
$$

since

$$
\begin{aligned}
\int_{M} \tilde{F}^{c} \frac{\partial \omega^{c}}{\partial y_{l}} g^{i j}\left(\Gamma \left(h_{i j}^{l}\right.\right. & \left.-\Gamma(g)_{i j}^{l}\right) d V_{g(t)} \leq \epsilon_{1} \int_{M}\left|\tilde{F}^{c}\right| d V_{g(t)} \\
\int_{M} \tilde{F}^{c} g^{i j} \pi_{a b}^{c} \tilde{F}_{i}^{a} \tilde{F}_{j}^{b} & \leq C\left(\int_{M} e(\tilde{F})^{2}\right)^{1 / 2}\left(\int_{M}\left(\tilde{F}^{c}\right)^{2}\right)^{1 / 2} \\
& \left.<C \epsilon \epsilon_{1}\left[\int\left(\tilde{F}^{c}\right)^{2}\right)\right]^{1 / 2}
\end{aligned}
$$

In the above estimates, we have used the energy estimates (4.5), the fact that $g(t) \rightarrow h$ as $t \rightarrow \infty$ uniformly on $M$ and that $|\tilde{F}|_{W^{2, N}}<\epsilon$ for $t \in\left[t_{0}, t_{0}+\delta\right)$ (which implies $|\tilde{F}|_{C^{k, \alpha}}<\tilde{\epsilon}$ for sufficiently big $N$ ). For those reasons, $\epsilon_{1}<<\epsilon$ is a constant that can be made much smaller than $\epsilon$, by taking $\epsilon$ small and $s_{0}$ big. Integrate (4.7) in $t$.

$$
\begin{aligned}
\frac{1}{2} \sup _{t \in\left[t_{0}, t_{0}+\delta\right)} \int\left(\tilde{F}^{c}\right)^{2}(t) d V_{g(t)} & +\sup _{t \in\left[t_{0}, t_{0}+\delta\right)} \int_{t_{0}}^{t} \int_{M}\left|\nabla \tilde{F}^{c}\right|^{2} d V_{g(t)} \\
& \leq \int \frac{1}{2}\left(\tilde{F}^{c}\right)^{2}\left(t_{0}\right) d V_{h}+C A \epsilon \epsilon_{1}
\end{aligned}
$$


Since for big $t_{0}$ the first integral on the right-hand side of the previous inequality can be made much smaller than $\epsilon$, it follows that for big $t_{0}$ and small $\epsilon$,

$$
\begin{aligned}
& \sup _{t \in\left[t_{0}, t_{0}+\delta\right)} \int_{M}\left(\tilde{F}^{c}\right)^{2}(t) d V_{g(t)}<\tilde{\epsilon}, \\
& \sup _{t \in\left[t_{0}, t_{0}+\delta\right)} \int_{M}\left|\nabla \tilde{F}^{c}\right|^{2} d V_{g(t)}<\tilde{\epsilon},
\end{aligned}
$$

for some constant $\tilde{\epsilon}<<\epsilon$ and these estimates depend on $A$.

Step 4.5. $\sup _{t \in\left[t_{0}, t_{0}+\delta\right)} \int_{t_{0}}^{t} \int_{M}\left|\frac{d}{d t} \tilde{F}^{c}\right|^{2} d V_{g(t)}$ and $\sup _{t \in\left[t_{0}, t_{0}+\delta\right)} \int_{t_{0}}^{t} \int_{M}\left|\nabla^{2} \tilde{F}^{c}\right|^{2}$ $d V_{g(t)}$ can be made much smaller than $\epsilon$ for big enough $s_{0}$ which depends on $A$ and on the rate of convergence of $g(t)$ to $h$, for small enough $\epsilon$.

$$
\frac{d}{d t} \tilde{F}^{c}=\Delta \tilde{F}^{c}+H^{c}, \text { where } H^{c}=\frac{\partial \omega^{c}}{\partial y_{l}} g^{i j}\left(\Gamma(h)_{i j}^{l}-\Gamma(g)_{i j}^{l}\right)+g^{i j} \pi_{a b}^{c} \tilde{F}_{i}^{a} \tilde{F}_{j}^{b} .
$$

Then,

$$
\begin{aligned}
\left(H^{c}\right)^{2} & =\left(\Delta \tilde{F}^{c}\right)^{2}+\left(\frac{d}{d t} \tilde{F}^{c}\right)^{2}-2 \Delta \tilde{F}^{c} \frac{d}{d t} \tilde{F}^{c} \\
-\int_{M} \frac{d}{d t} \tilde{F}^{c} \Delta \tilde{F}^{c}= & \int_{M} g^{i j} \nabla_{i}\left(\frac{d}{d t} \tilde{F}^{c}\right) \nabla_{j} \tilde{F}^{c} \\
= & \frac{1}{2} \frac{d}{d t} \int_{M}\left|\nabla \tilde{F}^{c}\right|^{2}+\frac{1}{2} \int_{M} g^{i p} g^{j q}\left(-2 R_{p q}+\frac{1}{\tau} g_{p q}\right)\left|\nabla \tilde{F}^{c}\right|^{2} \\
& -\frac{1}{2} \int_{M}\left|\nabla \tilde{F}^{c}\right|^{2}\left(\frac{n}{2 \tau}-R\right) . \\
\int_{M}\left(\Delta \tilde{F}^{c}\right)^{2} & =\int_{M}\left|\nabla^{2} \tilde{F}^{c}\right|^{2}+\int_{M} g^{i j} g^{k s} \nabla_{j} \tilde{F}^{c} R_{j k p}^{s} \nabla_{p} \tilde{F}^{c}
\end{aligned}
$$

Combining (4.8), (4.9) and (4.10), we get

$$
\begin{array}{r}
\int_{M}\left|\frac{d}{d t} \tilde{F}^{c}\right|^{2}+\int_{M}\left|\nabla^{2} \tilde{F}^{c}\right|^{2}+\frac{d}{d t} \int_{M}\left|\nabla \tilde{F}^{c}\right|^{2} \\
\leq \int_{M}\left(H^{c}\right)^{2}+C \int_{M}\left|\nabla \tilde{F}^{c}\right|^{2} d V_{g(t)}
\end{array}
$$


since

$$
\begin{gathered}
\int_{M} g^{i p} g^{j q}\left(2 R_{p q}-\frac{1}{\tau} g_{p q}\right)\left|\nabla \tilde{F}^{c}\right|^{2} \leq \epsilon_{1} \int\left|\nabla \tilde{F}^{c}\right|^{2}, \\
\int_{M} g^{i j} g^{k s} \nabla_{j} \tilde{F}^{c} R_{j k p}^{s} \nabla_{p} \tilde{F}^{c} \leq C \int_{M}\left|\nabla \tilde{F}^{c}\right|^{2}
\end{gathered}
$$

Notice also that

$$
\begin{aligned}
\int_{M}\left(H^{c}\right)^{2} d V_{g(t)} & \leq \int_{M} C\left(g^{i j}\left(\Gamma_{i j}^{l}(h)-\Gamma_{i j}^{l}(g)\right)\right)^{2} d V_{g(t)}+C \int_{M} e(\tilde{F})^{2} d V_{g(t)} \\
& <\epsilon_{1}+C \epsilon \epsilon_{1}
\end{aligned}
$$

since $e(\tilde{F})<\epsilon$ for $t \in\left[t_{0}, t_{0}+\delta\right)$ and $\int_{M} e(\tilde{F}) d V_{g(t)}<\epsilon_{1}$ by energy estimates (4.5). We will sometimes use the same constant $\epsilon_{1}$ to denote any constant that can be made much smaller than $\epsilon$, (the estimates above are possible if we start with $\epsilon$ small enough and increase $s_{0}$ if necessary, depending on how big $A$ is). If we integrate (4.11) in $t$ and use the above estimates, we get

$$
\begin{aligned}
\int_{M} & \left|\nabla \tilde{F}^{c}(t)\right|^{2} d V_{g(t)}+\int_{t_{0}}^{t} \int_{M}\left|\frac{d}{d t} \tilde{F}^{c}\right|^{2}+\int_{t_{0}}^{t} \int_{M}\left|\nabla^{2} \tilde{F}^{c}\right|^{2} \\
& \leq \int_{M}\left|\nabla \tilde{F}^{c}\left(t_{0}\right)\right|^{2} d V_{g\left(t_{0}\right)}+\int_{t_{0}}^{t} \int_{M}\left(H^{c}\right)^{2}+C \int_{t_{0}}^{t} \int_{M}\left|\nabla \tilde{F}^{c}\right|^{2} d V_{g(s)} d s \\
& \leq \epsilon_{1},
\end{aligned}
$$

because of Step 4.4, the fact that $\int_{M}\left|H^{c}\right|^{2}<\epsilon_{1}$ for big $t_{0}$ and the fact that for big $t_{0} \int_{M}\left|\nabla^{k} \tilde{F}\right|^{2}\left(t_{0}\right) d V_{g\left(t_{0}\right)}$ can be made very small.

Step 4.6. $\sup _{t \in\left[t_{0}, t_{0}+\delta\right)} \int_{M}\left|\frac{d}{d s} \tilde{F}^{c}\right|^{2}, \quad \int_{t_{0}}^{t} \int_{M}\left|\nabla \frac{d}{d t} \tilde{F}^{c}\right|^{2}$ can be made much smaller than $\epsilon$ for big $t_{0}$, for all $t \in\left[t_{0}, t_{0}+\delta\right)$.

Let $\hat{F}^{c}=\frac{d}{d t} \tilde{F}^{c}$. Then

$$
\frac{d}{d t} \hat{F}^{c}=\Delta \hat{F}^{c}+g^{i p} g^{j q}\left(2 R_{p q}-\frac{1}{\tau} g_{p q}\right) \nabla_{i} \nabla_{j} \tilde{F}^{c}+\frac{d}{d t} H^{c}-g^{i j} \frac{d}{d t} \Gamma_{i j}^{k} \nabla_{k} \tilde{F}^{c}
$$


Multiply this equation by $\hat{F}^{c}$ and integrate it over $M$.

$$
\begin{aligned}
\frac{1}{2} \frac{d}{d t} \int_{M}\left(\hat{F}^{c}\right)^{2} & -\frac{1}{2} \int_{M}\left(\hat{F}^{c}\right)^{2}\left(\frac{n}{2 \tau}-R\right)=-\int_{M}\left|\nabla \hat{F}^{c}\right|^{2} \\
& +\int_{M} g^{i p} g^{j q}\left(2 R_{p q}-\frac{1}{\tau} g_{p q}\right) \nabla_{i} \nabla_{j} \tilde{F}^{c} \hat{F}^{c} \\
& +\int_{M} \hat{F}^{c} \frac{d}{d t} H^{c}-\int_{M} g^{i j} \frac{d}{d t} \Gamma_{i j}^{k} \nabla_{k} \tilde{F}^{c} \hat{F}^{c} .
\end{aligned}
$$

Integrate it in $t$ to get

$$
\begin{aligned}
\frac{1}{2} \int_{M}\left(\hat{F}^{c}(t)\right)^{2}+ & \int_{t_{0}}^{t} \int_{M}\left|\nabla \hat{F}^{c}\right|^{2} \leq \frac{1}{2} \int_{M}\left(\hat{F}^{c}\left(t_{0}\right)\right)^{2}+\frac{1}{2} \int_{t_{0}}^{t} \int_{M}\left(\hat{F}^{c}\right)^{2}\left(\frac{n}{2 \tau}-R\right) \\
& +\epsilon_{1}\left(\int_{t_{0}}^{t} \int\left|\nabla^{2} \tilde{F}^{c}\right|^{2}\right)^{1 / 2}\left(\int_{t_{0}}^{t} \int\left|\hat{F}^{c}\right|^{2}\right)^{1 / 2} \\
& \left.+\left(\int_{t_{0}}^{t} \int_{M}\left(\hat{F}^{c}\right)^{2}\right)^{1 / 2}\left(\int_{t_{0}}^{t} \int_{M} \frac{d}{d t} H^{c}\right)^{2}\right)^{1 / 2} \\
& +C\left(\int_{t_{0}}^{t} \int_{M}\left|\nabla \tilde{F}^{c}\right|^{2}\right)^{1 / 2}\left(\int_{t_{0}}^{t} \int_{M}\left(\hat{F}^{c}\right)^{2}\right)^{1 / 2} .
\end{aligned}
$$

Notice that

$$
\begin{aligned}
\int_{t_{0}}^{t} \int_{M}\left(\frac{d}{d t} H^{c}\right)^{2} \leq & C\left(\int_{t_{0}}^{t} \int_{M}\left(\frac{d}{d t}\left(\frac{\partial \omega^{c}}{\partial y^{l}} g^{i j}\right)\left(\Gamma(h)_{i j}^{l}-\Gamma(g)_{i j}^{l}\right)\right)\right)^{2} \\
& +\int_{t_{0}}^{t} \int_{M}\left(\left(\frac{d}{d t} g^{i j}\right) \pi_{a b}^{c} \tilde{F}_{i}^{a} \tilde{F}_{j}^{b}\right)^{2} \\
& +\int_{t_{0}}^{t} \int_{M}\left(g^{i j} \pi_{a b}^{c}\left(\frac{d}{d t} \tilde{F}_{i}^{a}\right) \tilde{F}_{j}^{b}\right)^{2}
\end{aligned}
$$

where

$$
\begin{gathered}
\int_{t_{0}}^{t} \int_{M}\left(\frac{d}{d t}\left(g^{i j} \frac{\partial \omega^{c}}{\partial y^{l}}\left(\Gamma(h)_{i j}^{l}-\Gamma(g)_{i j}^{l}\right)\right)\right)^{2}<\epsilon_{1}, \\
\int_{t_{0}}^{t} \int_{M}\left(\left(\frac{d}{d t} g^{i j}\right) \pi_{a b}^{c} \tilde{F}_{i}^{a} \tilde{F}_{j}^{b}\right)^{2} \leq \epsilon_{1},
\end{gathered}
$$

if $t_{0}$ is big enough, since $g(t) \rightarrow h$ uniformly on $M$ and $\frac{d}{d t} g^{i j}=g^{p i} g^{q j}\left(2 R_{p q}-\right.$ $\left.\frac{1}{\tau} g_{p q}\right)$, and

$$
\int_{t_{0}}^{t} \int_{M}\left(g^{i j} \pi_{a b}^{c}\left(\frac{d}{d t} \tilde{F}_{i}^{a}\right) \tilde{F}_{j}^{b}\right)^{2} \leq C \epsilon \int_{t_{0}}^{t} \int_{M}\left|\nabla \hat{F}^{c}\right|^{2}<\frac{1}{2} \int_{t_{0}}^{t} \int_{M}\left|\nabla \hat{F}^{c}\right|^{2},
$$


if we choose $\epsilon$ small enough, such that $C \epsilon<\frac{1}{2}$, since $\left|\nabla_{j} \tilde{F}\right|<\epsilon$ for $t \epsilon$ $\left[t_{0}, t_{0}+\delta\right)$.

$$
\int_{M}\left|\hat{F}^{c}\right|^{2} d V_{g(t)} \leq C\left(\int_{t_{0}}^{t} \int_{M}\left|\nabla^{2} \tilde{F}^{c}\right|^{2}+C \int_{t_{0}}^{t_{0}+\delta} \int_{M}\left|\nabla \tilde{F}^{c}\right|^{2}+\epsilon_{1}\right) \leq C\left(\epsilon_{1}+\epsilon_{1}\right),
$$

by Step 4.5. The assertion of Step 4.6 follows now immediately from (4.14).

From the estimate (4.15), we can now get (using the estimates of Steps 4.4, 4.5 and 4.6) that $\int_{t_{0}}^{t} \int_{U}\left(\frac{d}{d t} H^{c}\right)^{2}$ can be much smaller than $\epsilon$. Consider the equation

$$
\frac{d}{d t} \hat{F}^{c}=\Delta \hat{F}^{c}+\hat{H}^{c}
$$

where $\hat{F}^{c}=\frac{d}{d t} \tilde{F}^{c}$ and

$$
\hat{H}^{c}=g^{i p} g^{j q}\left(2 R_{p q}-\frac{1}{\tau} g_{p q}\right) \nabla_{i} \nabla_{j} \tilde{F}^{c}+\frac{d}{d t} H^{c}-g^{i j} \nabla_{k} \tilde{F}^{c} \frac{d}{d t}\left(\Gamma_{i j}^{k}\right)
$$

. Since $g(t) \rightarrow h$, where $\operatorname{Ric}(h)=\frac{1}{2 \tau} h$, by using the previous estimates, we can easily see that $\int_{t_{0}}^{t} \int_{U}\left(\hat{H}^{c}\right)^{2} d V_{g(s)} d s$ can be made much smaller than $\epsilon$. In the same manner as we have obtained the estimates in step 4.5 for $\tilde{F}^{c}$, we can get the following estimates for $\hat{F}^{c}=\frac{d}{d t} \tilde{F}^{c}$ by considering the evolution equation (4.17).

$$
\begin{gathered}
\sup _{t \in\left[t_{0}, t_{0}+\delta\right)} \int_{U}\left|\nabla \frac{d}{d t} \tilde{F}^{c}\right|^{2} \\
\int_{t_{0}}^{t_{0}+\delta} \int_{U}\left|\nabla^{2} \frac{d}{d t} \tilde{F}^{c}\right|^{2} \\
\int_{t_{0}}^{t_{0}+\delta} \int_{U}\left(\frac{d^{2}}{d t^{2}} \tilde{F}^{c}\right)^{2}
\end{gathered}
$$

can be made much smaller than $\epsilon$ for big $t_{0}$.

We have that $\Delta \tilde{F}^{c}=\frac{d}{d t} \tilde{F}^{c}-H^{c}$ where $W^{1,2}$ norm of $\frac{d}{d t} \tilde{F}^{c}$ and $L^{2}$ norm of $H^{c}$ can be made much smaller than $\epsilon$. By elliptic regularity theory, we can get that $W^{2,2}$ norm of $\tilde{F}^{c}$ can be made much smaller than $\epsilon$ (since it can be estimated in terms of $W^{1,2}$ norm of $\frac{d}{d t} \tilde{F}^{c}$ and $L^{2}$ norm of $\left.H^{c}\right)$. Using that and the fact that $|\tilde{F}|_{1, \alpha, g}<\epsilon$ notice that 


$$
\begin{aligned}
\int_{M}\left|\nabla H^{c}\right|^{2}< & C\left(\epsilon_{1}+\int_{M} g^{r s}\left(\nabla_{r}\left(g^{i j} \pi_{a b}^{c}\right)\right) \tilde{F}_{i}^{a} \tilde{F}_{j}^{b}\right)\left(\nabla_{s} g^{i^{\prime} j^{\prime}} \pi_{a^{\prime} b^{\prime}}^{c^{\prime}} \tilde{F}_{i^{\prime}}^{a^{\prime}} \tilde{F}_{j^{\prime}}^{b^{\prime}}\right) \\
& +\int g^{r s}\left(g^{i j} \pi_{a b}^{c} \nabla_{r} \tilde{F}_{i}^{a} \tilde{F}_{j}^{b}\right)\left(g^{i^{\prime} j^{\prime}} \pi_{a^{\prime} b^{\prime}}^{c^{\prime}} \nabla_{s} \tilde{F}_{i^{\prime}}^{a^{\prime}} \tilde{F}_{j^{\prime}}^{b^{\prime}}\right) \\
< & C\left(\epsilon_{1}+\epsilon \sum_{a} \int_{M}\left|\nabla \tilde{F}^{a}\right|^{2}+\epsilon \sum_{b} \int_{M}\left|\nabla^{2} \tilde{F}^{b}\right|^{2}\right) \\
< & \tilde{\epsilon}
\end{aligned}
$$

for some small constant $\tilde{\epsilon}$, that can be assumed to be much smaller than $\epsilon$, since $W^{2,2}$ norm of $\tilde{F}^{c}$ can be made much smaller than $\epsilon$. By elliptic regularity theory this implies that $W^{3,2}$ norm of $\tilde{F}^{c}$ can be made much smaller than $\epsilon$ for $t_{0}$ very big.

We can continue our proof by studying the equation $\frac{d}{d t} \hat{F}^{c}=\Delta \hat{F}^{c}+\hat{H}^{c}$. $\left|\tilde{F}^{c}\right|_{2, \alpha}<\epsilon$ for $t \in\left[t_{0}, t_{0}+\delta\right)$. By a standard parabolic regularity we can get the higher order estimates of $\tilde{F}^{c}$, by constants that are comparable to $\epsilon$. Therefore, by the similar analysis as above, we can get that $W^{3,2}$ norms of $\hat{F}^{c}$ can be made much smaller than $\epsilon$, since from the estimates that we have got till this point, we can again easily get that $W^{1,2}$ norm of $\hat{H}^{c}$ can be made much smaller than $\epsilon$. Consider again the equation

$$
\Delta \tilde{F}^{c}=\frac{d}{d t} \tilde{F}^{c}-H^{c} .
$$

We know that $W^{3,2}$ norm of $\frac{d}{d t} \tilde{F}^{c}$ and $W^{3,2}$ norms of $\tilde{F}^{c}$ can be made much smaller than $\epsilon$. Let's check that $W^{3,2}$ norm of $H^{c}$ can be made much smaller than $\epsilon$ as well. In order for it to be true, it is enough to check that $\int_{M}\left|\nabla^{3}\left(g^{i j} \pi_{a b}^{c} \tilde{F}_{i}^{a} \tilde{F}_{j}^{b}\right)\right|^{2}$ can be made much smaller than $\epsilon$.

$$
\begin{aligned}
\int\left|\nabla^{3}\left(g^{i j} \pi_{a b}^{c} \tilde{F}_{i}^{a} \tilde{F}_{j}^{b}\right)\right|^{2} \leq & C\left(\epsilon \int_{M}|\nabla \tilde{F}|^{2}\right. \\
& +C \sum_{a, b} \int\left|\nabla^{4} \tilde{F}^{a}\right|^{2}\left|\nabla \tilde{F}^{b}\right|^{2} \\
& \left.+C \sum_{a, b} \int_{M}\left|\nabla^{3} \tilde{F}^{a}\right|^{2}\left|\nabla^{2} \tilde{F}^{b}\right|^{2}\right) \\
< & C\left(\epsilon_{1}+C \epsilon \epsilon_{1}+\epsilon_{1}^{2}\right)<\tilde{\epsilon}
\end{aligned}
$$


since $|\tilde{F}|_{W^{2, N}, g(t)}<\epsilon$ for all $t \in\left[t_{0}, t_{0}+\delta\right)$ and all $t_{0} \geq s_{0}$. From here, again by elliptic regularity theory applied to equation (4.18), it follows that $W^{5,2}$ norm of $\tilde{F}^{c}$ can be made much smaller than $\epsilon$.

We can continue the proof in a similar manner as above, by taking the higher order derivatives of our original equation $\Delta \tilde{F}^{c}=\frac{d}{d t} \tilde{F}^{c}-H^{c}$ in $t$, using the estimates that we get on the way and then go backward to our original equation to improve a regularity of $\tilde{F}^{c}$. As a result, we can get (performing the previously described procedure sufficiently many times) that $|\tilde{F}|_{W^{N, 2}, g(t)}<\epsilon$ continues to hold past time $t_{0}+\delta$.

So far, we have proved that for every $A>0$ and an integer $k$ there exists $\epsilon_{0}=\epsilon_{0}(A, k)$ such that for every $\epsilon<\epsilon_{0}$ we can find $s_{0}=s_{0}(A, \epsilon, k)$, so that $\forall t_{0} \geq s_{0}$ there exists a solution of

$$
\begin{aligned}
\frac{d}{d t} \phi(t) & =\Delta_{g(t), h} \phi(t) \\
\phi\left(t_{0}\right) & =\phi_{t_{0}}
\end{aligned}
$$

for all $t \in\left[t_{0}, t_{0}+A\right]$ and $|\phi-\mathrm{Id}|_{k, \alpha}<\epsilon$.

We want to show that these maps $\phi(t): M \rightarrow M$ are actually diffeomorphisms which will imply that we have constructed an 1- parameter family of gauges such that for $\bar{g}(t)=\left(\phi(t)^{*}\right)^{-1} g(t)$ the linearization of the Ricci-DeTurck flow

$$
\frac{d}{d t} \bar{g}=-2 \bar{R}_{i j}+\frac{1}{\tau} \bar{g}_{i j}+\nabla_{i} W_{j}+\nabla_{j} W_{i}
$$

with $\bar{g}\left(t_{0}\right)=\left(\phi_{t_{0}}^{-1}\right)^{*} g\left(t_{0}\right)$ is strictly parabolic $\left(W_{j}=\bar{g}_{j k} \bar{g}^{p q}\left(\Gamma_{p q}^{k}(\bar{g})-\Gamma(h)_{p q}^{k}\right)\right)$.

Corollary 4.7. Adopt the notation from Proposition 4.1. $\phi(t)$ are diffeomorphisms for all $t \in\left[t_{0}, t_{0}+A\right]$ and all $t_{0} \geq s_{0}$.

Proof. Fix any $t_{0} \geq s_{0}$. Consider the equation

$$
\begin{aligned}
\frac{d}{d t} \tilde{g}_{i j} & =-2 \tilde{R}_{i j}+\frac{1}{\tau} \tilde{g}_{i j}+\nabla_{i} V^{j}+\nabla_{j} V^{i}, \\
\tilde{g}\left(t_{0}\right) & =\left(\phi_{t_{0}}^{-1}\right)^{*} g\left(t_{0}\right),
\end{aligned}
$$


where $V^{k}=\tilde{g}^{p q}\left(\Gamma_{p q}^{k}(\tilde{g})-\Gamma_{p q}^{k}(h)\right)$. This is a strictly parabolic system of equations and therefore, there exists some $\delta>0$ so that a solution $\tilde{g}$ exists for all times $t \in\left[t_{0}, t_{0}+\delta\right)$. On the other hand, look at the system

$$
\begin{aligned}
\frac{d}{d t} \psi(t) & =-V \circ \psi(t), \\
\psi\left(t_{0}\right) & =\phi_{t_{0}} .
\end{aligned}
$$

Vector fields $V(t)$ are defined for $t \in\left[t_{0}, t_{0}+\delta\right)$ and therefore, the system (4.21) has a solution $\psi(t)$ for all those times. It is easy to show (a classical result) that all $\psi(t)$ are diffeomorphisms for $t \in\left[t_{0}, t_{0}+\delta\right)$. The simple computation (due to the fact that $g(t)$ is a solution of the Ricci flow equation) shows that

$$
\frac{d}{d t} \psi(t)=\Delta_{g(t), h} \psi(t)
$$

with $\psi\left(t_{0}\right)=\phi_{t_{0}}$. Because of the uniqueness of a harmonic map flow with the same initial data (we know that our solutions are smooth and uniformly bounded, so the uniqueness follows by the arguments of Eells and Sampson in [5]), we have that $\psi(t)=\phi(t)$ for all $t \in\left[t_{0}, t_{0}+\delta\right)$. This means $\phi(t)$ is a diffeomorphism for $t \in\left[t_{0}, t_{0}+\delta\right)$ and $\tilde{g}(t)=\left(\phi(t)^{-1}\right)^{*} g(t)$. We know that for all $t \in\left[t_{0}, t_{0}+A\right]$, for $t_{0}$ sufficiently big, we have that $|\phi(t)-\mathrm{Id}|_{k, \alpha, h}<\epsilon$. Therefore, $\left|\phi^{-1}-\mathrm{Id}\right|_{k, \alpha}$ can be made small which implies that $|\tilde{g}(t)-g(t)|$ can be made very small, comparable to $\epsilon$, for all $t \in\left[t_{0}, t_{0}+\delta\right)$. We want to extend a solution $\tilde{g}(t)$ of (4.20) all the way up to $t_{0}+A$. Since $|\tilde{g}(t)-h|<\tilde{\epsilon}$ and since our flow (4.20) is strictly parabolic, there exists $t_{1}=t_{1}(h, \tilde{\epsilon})$ so that for every $t \in\left[t_{0}, t_{0}+\delta\right)$, a solution to (4.20) exists for all times $s \in\left[t, t+t_{1}\right)$. That means, we can extend our solution past time $t_{0}+\delta$. Since our estimates on $|\tilde{g}(t)-h|$ for those times for which a solution $\tilde{g}(t)$ exists are independent of $\delta \leq A$, we can easily extend our solution all the way up to $t_{0}+A$, with $|\tilde{g}(t)-h|$ staying very small (comparable to $\epsilon$ ) for all $t \in\left[t_{0}, t_{0}+A\right]$. Existence of $\tilde{g}(t)$ for $t \in\left[t_{0}, t_{0}+A\right]$ gives that $\phi(t)$ stays a diffeomorphism for all times up to $t_{0}+A$, because it solves the equation (4.21). 


\subsection{The integrable case.}

The proofs in this subsection are motivated by those in [3], where Cheeger and Tian have considered the uniqueness problem of tangent cones under the assumption of integrability of one of the tangent cones and under some curvature and volume bounds.

Remark 4.8. So far, we have proved that if a limit $h$ is an Einstein metric (this assumption is not essential and will be removed later, it only stands for techical simplicity) then for every $A>0$ and an integer $k$ there exists $\epsilon_{0}=\epsilon_{0}(A, k)$ such that for every $\epsilon \leq \epsilon_{0}$ there exists $s_{0}=s_{0}(\epsilon, A, k)$ with the property that for every $t_{0} \geq s_{0}$ there is an 1-parameter family of diffeomorphisms $\phi(t)$ so that

1. $\phi^{-1}$ solves a harmonic map flow equation

$$
\begin{aligned}
\frac{d}{d t} \phi^{-1} & =\Delta_{g, h} \phi^{-1}, \\
\phi^{-1}\left(t_{0}\right) & =\phi_{t_{0}},
\end{aligned}
$$

where $\delta_{\phi_{t_{0}}^{*} h}\left(g\left(t_{0}\right)\right)=0$, for $t \in\left[t_{0}, t_{0}+A\right]$,

2. $\tilde{g}=\phi^{*} g$ solves strictly parabolic equation on $\left[t_{0}, t_{0}+A\right]$

$$
\frac{d}{d t} \tilde{g}=-2 \operatorname{Ric}(\tilde{g})+\frac{1}{\tau} \tilde{g}+\nabla_{i} V_{j}+\nabla_{j} V_{i}
$$

where $V^{i}=\tilde{g}^{p q}\left(\Gamma_{p q}^{i}(\tilde{g})-\Gamma_{p q}^{i}(h)\right)$. We will say that $\tilde{g}$ is in a standard form around $h$. We will denote by $P_{h_{0}}(\tilde{g})=\nabla_{i} V_{j}+\nabla_{j} V_{i}$.

3. $|\phi-\operatorname{Id}|_{k, \alpha}<\epsilon$.

4. $|\tilde{g}-h|_{k, \alpha}<\epsilon$.

From now on, we will simply write $\phi g$ instead of $\phi^{*} g$. By the assumptions of Theorem 1.1 there exists a limit soliton, say $h(t)$ which is integrable. There is a sequence $t_{i}$ such that $g\left(t_{i}+t\right) \rightarrow h(t)$ as $i \rightarrow \infty$ and

$$
R_{i j}+\nabla_{i} \nabla_{j} f-\frac{1}{2 \tau} h_{i j}(t)=0
$$


for some function $f$. From before, we know that $f(t)$ is a minimizer for $\mathcal{W}$ with respect to a metric $h(t)$, for every $t$. Let $\psi(t)$ be 1-parameter family of diffeomorphisms induced by a vector field $-\nabla f$. Then $h(t)=\psi^{*}(t) h_{0}$, where $h_{0}=h(0)$. Since $h_{0}=\left(\psi^{-1}\right)^{*} h(t)$, it satisfies the equation

$$
0=\frac{d}{d t} h_{0}=-2 \operatorname{Ric}\left(h_{0}\right)+\frac{1}{\tau} h_{0}-\mathcal{L}_{\psi_{*} \frac{d}{d t} \psi^{-1}} h_{0},
$$

From $\psi \circ \psi^{-1}=\mathrm{Id}$, by taking a time derivative, we see that $\psi^{*} \frac{d}{d t} \psi^{-1}+$ $\psi^{*} \mathcal{L}_{\frac{d}{d t}} \psi^{-1}=0$ and since $\psi$ is a diffeomorphism, we get that

$$
\frac{d}{d t} \psi^{-1}=-\mathcal{L}_{\frac{d}{d t} \psi} \psi^{-1}=\mathcal{L}_{\nabla f(\psi)} \psi^{-1}
$$

Since $\{f(t)\}_{0 \leq t<\infty}$ are the minimizers for $\mathcal{W}$, there are uniform $C^{k+2, \alpha}$ estimates on $f(t)$. Since $\frac{d}{d t} \psi=-\nabla f(\psi)$, there are uniform $C^{k+1, \alpha}$ bounds on $\psi$, for $t \in[0, B]$. This together with (4.23) yields $\left|\psi^{-1}\right|_{k, \alpha} \leq C(B)$, for $t \in[0, B]$. Let $\tilde{g}(t)=\psi^{-1} g(t)$. Then $\tilde{g}(t)$ satisfies the equation

$$
\frac{d}{d t} \tilde{g}=-2 \operatorname{Ric}(\tilde{g})+\frac{1}{\tau} \tilde{g}-\mathcal{L}_{\psi_{*} \frac{d}{d t} \psi^{-1}} \tilde{g},
$$

and

$$
\left|\tilde{g}\left(t_{i}+t\right)-h_{0}\right|_{k, \alpha} \leq\left|\psi^{-1}\right|\left|g\left(t_{i}+t\right)-h(t)\right| \leq C(B)\left|g\left(t_{i}+t\right)-h(t)\right| \rightarrow 0,
$$

when $i \rightarrow \infty$, uniformly on $M \times[0, B]$ (that implies $\tilde{g}\left(t_{i}+t\right) \rightarrow h_{0}$ uniformly on compact subsets of $M \times[0, \infty))$. The proof of Proposition 4.1, after minor modifications can be used to get the following result that tell us how to find an appropriate gauge in the case of convergence toward the solitons instead of Einstein metrics.

Theorem 4.9. For every $L>0$ and an integer $k$, there exists $\epsilon_{0}=\epsilon_{0}(L, k)$ such that for every $\epsilon<\epsilon_{0}$, we can find $i_{0}=i_{0}(L, \epsilon, k)$, so that whenever $i \geq i_{0}$ there is a gauge $\phi(t)$ on $M \times\left[t_{i}, t_{i}+L\right]$ such that $\phi g$ is in a standard form around $h_{0}$ (see Remark 4.11 below), $\left|\phi \tilde{g}-h_{0}\right|_{k, \alpha}<\epsilon$ and $|\phi-\mathrm{Id}|_{k, \alpha}<\epsilon$.

Definition 4.10. A limit soliton $h(0)$ is said to be integrable if for every solution a of a linearized deformation equation

$$
\left.\frac{d}{d u}\left(\operatorname{Ric}_{g_{u}}+\mathcal{L}_{\psi_{*} \frac{d}{d t} \psi^{-1}} g_{u}-\frac{1}{\tau}\left(g_{u}\right)_{i j}\right)\right|_{u=0}=0
$$


with $g_{0}=h_{0}$ there exists a path of solitons $h_{u}$, satisfying the soliton equation

$$
\operatorname{Ric}_{h_{u}}+\mathcal{L}_{\psi_{*} \frac{d}{d t} \psi^{-1}} h_{u}-\frac{1}{\tau}\left(h_{u}\right)_{i j}=0,
$$

with $u \in(-\epsilon, \epsilon)$ and $h_{0}=h(0)$ such that

$$
\left.\frac{d}{d u}\right|_{u=0} h_{u}=\mathbf{a}
$$

Remark 4.11. In the context of Theorem 1.1, to say that $\bar{g}(t)$ is in a standard form around $h_{0}$ means that $\bar{g}$ satisfies the following equation

$$
\frac{d}{d t} \bar{g}=-2 \operatorname{Ric}(\bar{g})+\frac{1}{\tau} \bar{g}+P_{h_{0}}(\bar{g})-\mathcal{L}_{\psi_{*} \frac{d}{d t} \psi^{-1}} \bar{g},
$$

where $P_{h_{0}}(\tilde{g})=\nabla_{i} V_{j}+\nabla_{j} V_{i}$ and $V^{k}=\tilde{g}^{p q}\left(\Gamma_{p q}^{k}(\tilde{g})-\Gamma_{p q}^{k}\left(h_{0}\right)\right)$. We will write $h_{0}$ for $h(0)$ in a further discussion.

Choose $i_{0}, \phi$ as in Theorem 4.9 with $3 L$ instead of $L$. Denote by $\|\cdot\|_{a, b}=$ $\int_{a}^{b}|\cdot|$, where $|\cdot|$ is just the $L^{2}$ norm. Let $\pi$ denote an orthogonal projection on the subspace $\operatorname{ker}\left(-\frac{d}{d t}+\Delta+\frac{1}{\tau}+U\right)_{M \times\left[t_{i_{0}}, t_{i_{0}}+L\right]}$, with respect to norm $\|\cdot\|_{t_{i_{0}}, t_{i_{0}}+L}$, where $U$ is a linear first-order expression that comes out after linearizing the equation (4.25). Let $g_{1}$ be a suitable chosen soliton. Denote by $k=\phi g-g_{1}$ and put $\pi k=(\pi k)_{\uparrow}+(\pi k)_{\downarrow}+(\pi k)_{0}$. The integrability assumption on $h_{0}$ enters when we choose $g_{1}$ so that $(\pi k)_{0}=0$. Look at the explanation for $(\cdot)_{\uparrow},(\cdot)_{\downarrow}$ and $(\cdot)_{0}$, just after the equation (4.27) below.

Lemma 4.12. Let $h_{0}$ be an integrable limit soliton. Then if $\tau<\tau(n, L)$, for any cylinder $M \times\left[t_{i_{0}}, t_{i_{0}}+L\right]$ there is a soliton $g_{1}$ satisfying $P_{h_{0}}\left(g_{1}\right)=0$ and equation (4.24), and such that $(\pi k)_{0}=0$. Moreover, if

$$
\sup _{\left[t_{i_{0}}, t_{i_{0}}+L\right]}\left|\phi g(t)-h_{0}\right|<\tau
$$

then

$$
\left\|g_{1}-h_{0}\right\|_{t_{i_{0}}, t_{i_{0}}+L} \leq 2\left\|\pi\left(\phi g(t)-h_{0}\right)\right\|_{t_{i_{0}}, t_{i_{0}}+L}
$$

where the left-hand side of estimate (4.26) is time independent. 
Proof. The proof of this lemma follows the proof of Lemma 5.56 in [3]. The integrability assumption implies that the set of metrics $\tilde{g}$ satisfying

$$
\begin{gathered}
\operatorname{Ric}(\tilde{g})-\frac{1}{\tau} \tilde{g}+\mathcal{L}_{\psi_{*} \frac{d}{d t} \psi^{-1}} \tilde{g}=0, \\
P_{h_{0}}(\tilde{g})=0
\end{gathered}
$$

has a natural smooth manifold structure near $h_{0}$. Let $\mathcal{V}$ be a sufficiently small Euclidean neighborhood of $h_{0}$. The tangent space to $\mathcal{V}$ at $h_{0}$ is naturally identified with

$$
\mathcal{K}=\left\{a \in \operatorname{ker}\left(\Delta+\frac{1}{\tau}+U\right) \mid D P_{h_{0}} a=0\right\} .
$$

Define $\psi: \mathcal{V} \rightarrow \mathcal{K}$ by

$$
\psi(\tilde{g})=\sum_{i}\left\langle\tilde{g}, B_{i}\right\rangle B_{i}
$$

where $B_{i}$ is an orthonormal basis for $\mathcal{K}$ with respect to a natural inner product. $\psi$ is a smooth map and the differential of $\psi$ is the identity map. We can use now the implicit function theorem and Lemma 4.13 to finish the proof of the Lemma 4.12 .

The inequality (4.26) implies that $\left|g_{1}-h_{0}\right| \leq 2 \sup _{\left[t_{i_{0}}, t_{i_{0}}+L\right]} \mid \pi\left(\phi g(t)-h_{0} \mid\right.$, where $|\cdot|$ is just the usual $L^{2}$ norm. The linearization of the right-hand side of the equation $\frac{d}{d t} \phi g=Q(\phi g)$, satisfied by $\phi g$, where $\phi$ is a gauge chosen as in Theorem 4.9 is $D Q(k)=\Delta k+\frac{1}{\tau} k+U$, where $U$ is a linear first-order expression in $k$ and a Laplacian and $U$ are with respect to metric $\phi g$. Let $F$ be a solution of

$$
\frac{d}{d t} F=\mathcal{L} F
$$

where $\mathcal{L}=\Delta+\frac{1}{\tau}+U$ and the Laplacian and $U$ are this time given with a respect to a fixed metric (in our case, we will take metric $h_{0}$ ). Let $\left\{\lambda_{k}\right\}$ be the set of eigenvalues of $\mathcal{L}$. We can write $F=F_{\uparrow}+F_{\downarrow}+F_{0}$, where $F_{\uparrow}(t)=\sum_{\lambda_{k}<0} a_{k} e^{-\lambda_{k} t}, F_{\downarrow}(t)=\sum_{\lambda_{k}>0} a_{k} e^{-\lambda_{k} t}$, and $F_{0}$ is a projection of $F$ to a kernel of $\mathcal{L}$.

The basic parabolic estimates (for example similarly as in [15] and [3]) yield the following. 
Lemma 4.13. There exists $\tau>0$ such that for any solution $\eta$ of (4.27) with $\left|g_{1}-h_{0}\right|_{k+2, \alpha} \leq \tau$, we have that

$$
\sup _{\left(t_{0}, t_{0}+L\right)}|\eta|_{k, \alpha} \leq C \sup _{\left(t_{0}, t_{0}+L\right)}|\eta|
$$

where the first norm is $C^{k, \alpha}$ norm and the last norm is $L^{2}$ norm.

Lemma 4.14. There exists $\alpha>1$ such that

$$
\begin{gathered}
\sup _{[L, 2 L]}\left|F_{\uparrow}\right| \geq \alpha \sup _{[0, L]}\left|F_{\uparrow}\right|, \\
\sup _{[L, 2 L]}\left|F_{\downarrow}\right| \leq \alpha^{-1} \sup _{[0, L]}\left|F_{\downarrow}\right| .
\end{gathered}
$$

The norms considered above are standard $L^{2}$ norms.

Proof. We will prove only (4.28), since the proof of (4.29) is similar. Let $\delta=\min \left\{\left|\lambda_{k}\right| \neq 0\right\}>0$.

$$
\begin{aligned}
\sup _{[L, 2 L]}\left|F_{\uparrow}\right|-\alpha \sup _{[0, L]}\left|F_{\uparrow}\right| & =\sup _{[0, L]} \sum_{\lambda_{k}<0} a_{k}^{2} e^{-2 \lambda_{k} t} e^{-2 \lambda_{k} L}-\alpha \sup _{[0, L]} \sum_{\lambda_{k}<0} a_{k}^{2} e^{-2 \lambda_{k} t} \\
& \geq \sup _{[0, L]} \sum_{\lambda_{k}<0} a_{k}^{2} e^{-2 \lambda_{k} t}\left(e^{2 \delta L}-\alpha\right)
\end{aligned}
$$

which is positive, if $e^{2 \delta L}>\alpha$. We can choose $\alpha=e^{\delta L}>1$.

Lemma 4.15. There exists $\beta<\alpha$ such that if

$$
\sup _{[L, 2 L]}|F| \geq \beta \sup _{[0, L]}|F|
$$

then

$$
\sup _{[2 L, 3 L]}|F| \geq \beta \sup _{[L, 2 L]}|F|
$$

and if

$$
\sup _{[2 L, 3 L]}|F| \leq \beta^{-1} \sup _{[L, 2 L]}|F|
$$


then

$$
\sup _{[L, 2 L]}|F| \leq \beta^{-1} \sup _{[0, L]}|F|
$$

Moreover, if $F_{0}=0$ at least one of (4.31), (4.33) holds.

The proof of Lemma 4.15 is almost the same to the proof of analogous lemma (5.31) in [3]. We can choose $\beta$ to be of order $e^{\frac{L \delta}{4}}$.

Let $\eta=\phi g-g_{1}$, where $\phi$ is chosen as in Theorem 4.9 and $g_{1}$ is a soliton as in Lemma 4.12 which does not depend on $t$ for a considered time interval of length $L$.

\section{Lemma 4.16.}

$$
\frac{d}{d t}\left(\phi g-g_{1}\right)=\Delta_{h_{0}}\left(\phi g-g_{1}\right)+\frac{1}{\tau}\left(\phi g-g_{1}\right)+F\left(\phi g, h_{0}, g_{1}\right)+U\left(\phi g-g_{1}\right)
$$

where $\left|F\left(\phi g, h, g_{1}\right)\right|_{k, \alpha} \leq C\left(\left|g_{1}-h_{0}\right|+|\eta|_{k, \alpha}\right)\left|\nabla^{2} \eta\right|_{k-2, \alpha}+C\left(\mid \nabla\left(g_{1}-\right.\right.$ $\left.\left.h_{0}\right)\left.\right|_{k-1, \alpha}+|\nabla \eta|_{k-1, \alpha}\right)|\nabla \eta|_{k-1, \alpha}$ and $U$ is a first order linear expression in $\phi g-g_{1}$.

Proof. Since both $\phi g$ and $g_{1}$ are in a standard form around $h_{0}$ (recall that $\left.P_{h_{0}}\left(g_{1}\right)=0\right)$, by using a formula for linearization of a second order operator $-2 \operatorname{Ric}(\phi g)+P_{h_{0}}(\phi g)$, we get

$$
\begin{aligned}
\frac{d}{d t}\left(\phi g-g_{1}\right)= & \left(-2 \operatorname{Ric}(\phi g)+P_{h_{0}}(\phi g)-\mathcal{L}_{\phi_{*} \psi_{*} \frac{d}{d t} \psi^{-1}} \phi g\right)- \\
& -\left(-2 \operatorname{Ric}\left(g_{1}\right)+P_{h_{0}}\left(g_{1}\right)-\mathcal{L}_{\psi_{*} \frac{d}{d t} \psi} g_{1}\right)+\frac{1}{\tau}\left(\phi g-g_{1}\right) \\
= & \Delta_{\phi g}\left(\phi g-g_{1}\right)+\frac{1}{\tau}\left(\phi g-g_{1}\right)+U\left(\phi g-g_{1}\right)+\tilde{F}\left(\phi g, g_{1}\right),
\end{aligned}
$$

where $\left|\tilde{F}\left(\phi g, g_{1}\right)\right|_{k, \alpha} \leq C\left(|\eta|_{k, \alpha}\left|\nabla^{2} \eta\right|_{k-2, \alpha}+|\nabla \eta|_{k, \alpha}^{2}\right)$, by a similar computation to a computation in [3]. Furthermore, $\Delta_{\phi g} \eta=\Delta_{h_{0}} \eta+\left(\Delta_{\phi g}-\Delta_{h_{0}}\right) \eta$ and since $\left|\phi g-h_{0}\right|_{k, \alpha} \leq C\left(|\eta|_{k, \alpha}+\left|g_{1}-h_{0}\right|_{k, \alpha}\right)$, we have that $\left|\left(\Delta_{\phi g}-\Delta_{h_{0}}\right) \eta\right| \leq$ $C\left(|\eta|_{k, \alpha}+\left|g_{1}-h_{0}\right|_{k, \alpha}\right)\left|\nabla^{2} \eta\right|_{k, \alpha}$. The Lemma 4.16 now follows.

We assume that $\left|g_{1}-h_{0}\right|_{k, \alpha}<\epsilon$. Let $k$ be a solution to (4.34). Then, we have the following Proposition. 
Proposition 4.17. There exists $\epsilon_{0}>0$, depending on the uniform bounds on the geometries $g(t)$, such that if $\epsilon<\epsilon_{0}$, then if

$$
\sup _{[L, 2 L]}|k| \geq \beta \sup _{[0, L]}|k|
$$

then

$$
\sup _{[2 L, 3 L]}|k| \geq \beta \sup _{[L, 2 L]}|k|
$$

and if

$$
\sup _{[2 L, 3 L]}|k| \leq \beta^{-1} \sup _{[L, 2 L]}|k|
$$

then

$$
\sup _{[L, 2 L]}|k| \leq \beta^{-1} \sup _{[0, L]}|k|
$$

Moreover, if $(\pi k)_{0}=0$, at least one of (4.37), (4.39) holds.

Proof. Assume there exist a sequence of gauges $\phi_{i}$ and constants $\tau_{i} \rightarrow 0$, such that $\left|\eta_{i}\right|_{k, \alpha}=\left|\phi_{i} g-h\right|_{k, \alpha} \leq \tau_{i} \rightarrow 0$, but for which none of the assertions in Proposition 4.17 holds. Let $\psi_{i}=\frac{\eta_{i}}{\sup _{[L, 2 L]}\left|\eta_{i}\right|}$. Then in view of Lemma 4.13, from standard compactness results (as in [3]), we get that for a subsequence $\psi_{i} \stackrel{C^{k, \alpha}}{\rightarrow} \psi$ and

$$
\frac{d}{d t} \psi=\Delta_{h} \psi+U(\psi)+\frac{1}{\tau} \psi
$$

where $\psi$ has a property that contradicts Lemma 4.15 . Recall that $\beta$ is of order $e^{\frac{\epsilon L}{4}}$.

Proof of Theorem 1.1. We will adopt the notation from above. Take some $L>0$ big enough (we will see later how big we want to make it) and choose $\epsilon_{0}>0$ as in Theorem 4.9 so that the Theorem holds for $\epsilon_{0}$, and $3 L$. For every $\epsilon<\epsilon_{0}$ there exists $i_{0}$ such that for every $i \geq i_{0}$ there exists a gauge $\phi$ so that $\phi$ satisfies all the conditions in Theorem 4.9 , that is $\phi g$ is in a standard 
form around $h_{0},\left|\phi g-h_{0}\right|_{k, \alpha}<\epsilon$ and $\left|\frac{d}{d t} \phi g\right|<\tilde{\epsilon}$ on $M \times\left[t_{i}, t_{i}+3 L\right]$, where $\tilde{\epsilon}$ is comparable to $\epsilon$. For each $t_{i}$ pick up the largest possible $L^{\prime}$ (we will omit emphasizing a dependence of $L^{\prime}$ on $i$ and we will call it just $L^{\prime}$, since it is irrelevant for further discussion) such that $\phi$ is defined on $M \times\left[t_{i}, t_{i}+L^{\prime}\right)$, $\phi g$ is in a standard form around $h$ and $|\phi g-h|_{k, \alpha}<\epsilon$ and $\min _{\left[t_{i}, t_{i}+3 L\right]} \mid \phi g-$ $\left.h\right|_{k, \alpha}<\frac{\epsilon}{1000}$. Divide $\left[t_{i}, t_{i}+L^{\prime}\right)$ into the subintervals of length $L$ and assume that $N$ is the largest number such that $\left[t_{i}+(N-1) L, t_{i}+N L\right] \subset\left[t_{i}, t_{i}+L^{\prime}\right)$.

Notice that for $L$ chosen above, from the proof of Theorem 4.9, all the estimates that we have got on $|\phi-\mathrm{Id}|_{k, \alpha}$ in the previous subsection depend on a polynomial in $L$ (call it $q(L)$ ), whose coefficients depend only on a dimension, an integer $k$ and the uniform bounds on geometries $g(t)$. By the estimates established in Proposition 4.1, we can increase $i_{0}$ if necessary, so that

1. For every $i \geq i_{0}$, we can find a gauge on $M \times\left[t_{i}, t_{i}+3 L\right]$, such that $\sup _{\left[t_{i}, t_{i}+3 L\right]}\left|\phi g(t)-h_{0}\right|_{k, \alpha}<\frac{\epsilon}{1000 e^{L \delta / 4}}$.

2. If the initial data $\phi(s)$ is such that $|\phi(s)-\operatorname{Id}|_{k, \alpha}<\frac{\epsilon}{e^{L \delta / 8}}$ and $\left|\phi(s)^{*} g(s)-h_{0}\right|_{k, \alpha}<\frac{\epsilon}{e^{L \delta / 8}}$, where $s \in\left[t_{i}, t_{i}+L^{\prime}\right]$, for $i \geq i_{0}$, then $\phi$ can be extended to interval $[s, s+3 L]$ such that $\sup _{[s, s+3 L]}\left|\phi g-h_{0}\right|_{k, \alpha}<$ $\frac{\epsilon}{100 p(L)}$ (we might need increase $i_{0}$ for this to hold). Polynomial $p(L)$ can be any polynomial with leading coefficient 1 and with a degree that is e.g. one more than a degree of $q(L)$.

3. If the initial data is such that $|\phi(s)-\operatorname{Id}|_{k, \alpha}<\frac{\epsilon}{p(L)}$ and $\mid \phi(s)^{*} g(s)-$ $\left.h_{0}\right|_{k, \alpha}<\frac{\epsilon}{p(L)}$, where $s \in\left[t_{i}, t_{i}+L^{\prime}\right]$, for $i \geq i_{0}$, then $\phi$ can be extended on interval $[s, s+3 L]$ such that $\sup _{[s, s+3 L]}\left|\phi g-h_{0}\right|_{k, \alpha}<\epsilon$.

We want to show that there exists $i$ (for sufficiently big $L$, so that, above holds) such that a corresponding $L^{\prime}=\infty$. Assume that for all $i \geq i_{0}$ and all $\epsilon>0, L^{\prime}<\infty$. Denote by $I_{j}=\left[t_{i}+j L, t_{i}+j L+L\right]$. Assume that $\epsilon$ is small enough so that we can apply Lemma 4.12, that is for every $j$ there exists a soliton $g_{j}$ such that $\left(\pi\left(\phi g-g_{j}\right)\right)_{0}=0$ on $I_{j}$ and therefore by Proposition 4.17, $\phi g-g_{j}$ either satisfies a growth condition $((4.36) \Rightarrow(4.37))$ or a decay 
condition $((4.38) \Rightarrow(4.39))$. Moreover, $\left|g_{j}-h_{0}\right| \leq 2 \sup _{I_{j}}\left|\pi\left(\phi g-h_{0}\right)\right| \leq$ $C \sup _{I_{j}}\left|\phi g-h_{0}\right|_{k, \alpha}$. We need to consider two cases.

Case 4.18. Assume that for all $i_{0}$ and all $i \geq i_{0}$, where $i_{0}=i_{0}(L)$ is chosen as in Theorem 4.9 for L big enough (so that (1), (2) and (3) hold), and for all the intervals $I_{j}$ (that are defined with respect to $t_{i}$; we want to omit double indices) for which we have $\sup _{I_{j}}\left|\phi g-h_{0}\right|_{k, \alpha} \leq \frac{\epsilon}{100 p(L)}, \phi g-g_{j}$ satisfies a decay condition on $I_{j}$ (recall that $L^{2}$ norms are considered in a growth and a decay condition).

By using Proposition 4.17 inductively, we get that

$$
\sup _{I_{l}}\left|\phi g-g_{j}\right| \leq \frac{1}{\beta^{l}} \sup _{I_{1}}\left|\phi g-g_{j}\right|
$$

for all $l \leq j$. Moreover, $\sup _{I_{1}}\left|\phi g-g_{j}\right| \leq \sup _{I_{1}}\left|\phi g-h_{0}\right|+\left|g_{j}-h_{0}\right| \leq$ $\sup _{I_{1}}\left|\phi g-h_{0}\right|+2 \sup _{I_{j}}\left|\phi g-h_{0}\right|<\frac{3 \epsilon}{100 p(L)}$, which yields

$$
\sup _{I_{l}}\left|\phi g-g_{j}\right| \leq \frac{1}{\beta^{l}} \frac{3 \epsilon}{100 p(L)}
$$

By Lemma 4.13, we may assume that $\sup _{I_{l}}\left|\phi g-g_{j}\right|_{k+2, \alpha} \leq \frac{\epsilon}{\beta^{l}}$. Whenever, we increase $L$ (the necessity for $L$ being increased will depend only on the uniform estimates), we can choose an appropriate $\epsilon_{0}$ as in Theorem 4.9 and take any $\epsilon<\epsilon_{0}$. Each time, we do that we might have to increase $i_{0}$ (depending on $\epsilon<\epsilon_{0}$ ). Therefore, on $M \times I_{l}$, for $l \leq j$, we have

$$
\begin{aligned}
\left|\frac{d}{d t} \phi g\right|_{k, \alpha}= & \left|\frac{d}{d t}\left(\phi g-g_{j}\right)\right|_{k, \alpha} \\
= & \left(-2 \operatorname{Ric}(\phi g)+2 \operatorname{Ric}\left(g_{j}\right)\right)+\frac{1}{\tau}\left(\phi g-g_{j}\right)+\left(P_{h_{0}}(\phi g)-P_{h_{0}}\left(g_{j}\right)\right) \\
& +\mathcal{L}_{\psi_{*} \frac{d}{d t} \psi^{-1}}\left(g_{j}-\phi g\right) \\
\leq & C \sup _{I_{l}}\left|\phi g-g_{j}\right|_{k+2, \alpha}<\frac{C \epsilon}{\beta^{l}}
\end{aligned}
$$


For every $l \leq j$, since $\frac{d}{d t} \phi g=\frac{d}{d t}\left(\phi g-h_{0}\right)$, we have that

$$
\begin{aligned}
\sup _{I_{l}}\left|\phi g-h_{0}\right|_{k, \alpha} & \leq 2 L \sup _{I_{l} \cup I_{l-1}}\left|\frac{d}{d t} \phi g\right|_{k, \alpha}+\sup _{I_{l-1}}\left|\phi g-h_{0}\right|_{k, \alpha} \\
& \leq 2 L C \frac{\epsilon}{\beta^{l-1}}+2 L C \frac{\epsilon}{\beta^{l-2}}+\cdots+2 L C \frac{\epsilon}{\beta}+\sup _{I_{2}}\left|\phi g-h_{0}\right|_{k, \alpha} \\
& \leq \sup _{I_{2}}\left|\phi g-h_{0}\right|_{k, \alpha}+\frac{2 L C \epsilon}{\beta-1}
\end{aligned}
$$

which can be made smaller than $\frac{\epsilon}{e^{L \delta / 8}}$ for $L$ chosen big enough at the beginning. By Condition 2, for big values of $i$, we can extend $\phi$ on $I_{j+1}$ so that $\sup _{I_{j+1}}\left|\phi g-h_{0}\right|<\frac{\epsilon}{100 p(L)}$ and it has to coincide with our previously constructed $\phi$ on $I_{j+1}$. We can continue a described procedure by looking now at intervals $I_{j}$ and $I_{j+1}$ replaced by intervals $I_{j+1}$ and $I_{j+2}$ respectively. If we repeat this sufficiently many times, we will reach the interval $I_{N-1}$ with

$$
\sup _{I_{N-1}}\left|\phi g-h_{0}\right|_{k, \alpha}<\frac{\epsilon}{100 p(L)} .
$$

By Condition 3, we will now be able to extend $\phi$ (for sufficiently big values of $i)$ to interval $\left[t_{i}+(N-1), t_{i}+(N+1) L\right]$, with $\sup _{\left[t_{i}+(N-1), t_{i}+(N+1) L\right]} \mid \phi g-$ $h_{0} \mid<\epsilon$ holding. Since $(N+1) L>L^{\prime}$, this estimate contradicts a maximality of $L^{\prime}$ with properties. Therefore, either there exists $i$ such that a corresponding $L^{\prime}=\infty$, or we have a following case holding.

Case 4.19. There are some $L, i$ and $j$ for which $\sup _{I_{j}}\left|\phi g-h_{0}\right|_{k, \alpha}<\frac{\epsilon}{100 p(L)}$, and $\phi g-h_{0}$ satisfies a growth condition on $I_{j}\left(I_{j}\right.$ is defined with respect to $\left.t_{i}\right)$.

By using Proposition 4.17 inductively, we would have that 


$$
\begin{aligned}
\sup _{I_{N-1}}\left|\phi g-g_{j}\right| & <\frac{1}{\beta} \sup _{I_{N}}\left|\phi g-g_{j}\right| \\
& \leq \frac{1}{\beta}\left(\sup _{I_{N}}\left|\phi g-h_{0}\right|+\left|g_{j}-h_{0}\right|\right) \\
& \leq \frac{1}{\beta}\left(\sup _{I_{N}}\left|\phi g-h_{0}\right|+2 \sup _{I_{j}}\left|\phi g-h_{0}\right|\right) \\
& <\frac{3 \epsilon}{\beta} .
\end{aligned}
$$

Moreover, if we use Lemma 4.13, together with the estimate

$$
\begin{aligned}
\sup _{I_{N-1}}\left|\phi g-h_{0}\right| & \leq \sup _{I_{N-1}}\left|\phi g-g_{j}\right|+\left|g_{j}-h_{0}\right| \\
& \leq \sup _{I_{N-1}}\left|\phi g-g_{j}\right|+2 \sup _{I_{j}}\left|\phi g-h_{0}\right| \\
& <\frac{3 \epsilon}{\beta}+\frac{\epsilon}{100 p(L)},
\end{aligned}
$$

which can be made smaller than $\frac{\epsilon}{p(L)}$, by Condition 3 we can extend $\phi$ to an interval $\left[t_{i}+(N-1) L, t_{i}+(N+1) L\right]$ (if $i$ is big enough), with $\left|\phi g-h_{0}\right|_{k, \alpha}<\epsilon$ holding. We again get a contradiction as in the previous case if we assume $L^{\prime}<\infty$ for all $i$.

Therefore, there exists $i_{0}$ such that a gauge $\phi$ can be constructed on $M \times\left[t_{i_{0}}, t_{i_{0}}+L^{\prime}\right)$, satisfying properties and such that a corresponding $L^{\prime}=\infty$. Consider again $I_{j}=\left[t_{i_{0}}+j L, t_{i_{0}}+j L+L\right]$ and the corresponding $g_{j}$ that are found by Lemma 4.12, such that for $k_{j}=\phi g-g_{j}$, we have that $\left(\pi k_{j}\right)_{0}=0$ on $M \times I_{j}$. Notice that a decay condition $((4.38 \Rightarrow(4.39))$ holds for all $j$. If there existed some $j$ for which it were not true, by using Proposition 4.17 inductively and standard parabolic estimates (Lemma 4.13), we would find that

$$
\epsilon>\sup _{\left[t_{i_{0}}+(N-1) L, t_{i_{0}}+N L\right]}\left|\phi g-g_{j}\right| \geq \beta^{N-j} \sup _{I_{j}}\left|\phi g-g_{j}\right|,
$$

for all $N$ and we would get a contradiction by letting $N$ tend to infinity (if $\sup _{I_{j}}\left|\phi g-g_{j}\right|=0$, our metric $g\left(t_{i_{0}}+j L\right)$ would be a soliton satisfying 
(4.22) and it would stay so for all later times which is not an interesting case). This means, we have a decay for all times if we do not start with a soliton.

After passing to a subsequence, we may assume that for some metric $g_{\infty}$ that satisfies a soliton type equation $\lim _{j \rightarrow \infty}\left|g_{j_{p}}-g_{\infty}\right|_{k, \alpha^{\prime}}=0$, where $\alpha^{\prime}<\alpha$.

Claim 4.20. $\lim _{p \rightarrow \infty} \sup _{I_{j_{p}}}\left|k_{j_{p}}\right|_{k, \alpha}=0$.

Proof. If it were not the case, there would exist a subsequence of $j_{p}$ (denote it by the same symbol) such that $\phi g-g_{j_{p}}$ would satisfy a growth condition, that is

$$
\sup _{\left[t_{i_{0}}+(N-1) L, t_{i_{0}}+N L\right]}\left|\phi g-g_{j_{p}}\right| \geq \beta^{\left(N-j_{p}\right)} \sup _{I_{j_{p}}}\left|\phi g-g_{j_{p}}\right|,
$$

for all $N$, where $\beta$ can be taken to be $e^{\frac{L \delta}{4}}$ and by taking $N \rightarrow \infty$ we immediately get a contradiction, since $\sup _{\left[t_{i_{0}}+(N-1) L, t_{i_{0}}+N L\right]}\left|\phi g-g_{j_{p}}\right|<C \epsilon$.

As in the proof of the claim above, we get that $\phi g-g_{j_{p}}$ has to satisfy a decay condition for all $p$. By Claim 4.20, by using Proposition 4.17 inductively and by standard parabolic estimates (Lemma 4.13), we find that for some $c>0$,

$$
\left|\phi g-g_{\infty}\right|_{k, \alpha} \leq c e^{-\frac{\delta\left(t-t_{i_{0}}\right)}{4}},
$$

for $t \in\left[t_{i_{0}}+(N-1) L, t_{i_{0}}+N L\right]$ and for all $N>0$, that is

$$
\left|\phi g-g_{\infty}\right|_{k, \alpha} \leq C e^{-c t}
$$

for all $t \geq t_{i_{0}}$. (4.40) implies that $\left|g(t)-\phi^{-1} g_{\infty}\right|_{C^{0}}<C e^{-c t} \cdot \phi^{-1} g_{\infty}$ is a soliton that moves by diffeomorphisms $\phi(t)^{-1}$ and therefore, is determined by metric $\phi^{-1}\left(t_{i_{0}}\right) g_{\infty}$. Since $h_{0}$ is a limit soliton of metrics $g\left(t_{i}\right), h_{0}$ and $\phi^{-1}\left(t_{i_{0}}\right) g_{\infty}$ differ only by a diffeomorphism, that is $\eta \phi^{-1}\left(t_{i_{0}}\right) g_{\infty}=h_{0}$ for some diffeomorphism $\eta$. Let finally $\phi^{\prime}=\eta \phi^{-1}\left(t_{i_{0}}\right) \phi(t)$. Then,

$$
\left|\phi^{\prime} g(t)-h_{0}\right|_{k, \alpha}<C e^{-c t}
$$


that is $\phi^{\prime} g(t)$ converges to a soliton $h_{0}$ exponentially as $t \rightarrow \infty$. We know that $h(t)=\psi(t) h_{0}$ and therefore,

$$
\left|\psi \phi^{\prime} g(t)-h(t)\right|_{C^{0}} \leq C e^{-c t}
$$

This finishes the proof of Theorem 1.1.

\section{Acknowledgments.}

I would like to thank my advisor Gang Tian for bringing this problem to my attention, for many useful discussions, suggestions and his constant support. Richard Hamilton, Huai-Dong Cao, Tom Ilmanen, Peter Topping and Jeff Viaclovsky deserve many thanks as well.

\section{References.}

[1] B. Kleiner, J. Lott, Notes on Perelman's paper (available at http://www.math.lsa.umich.edu/research/ricciflow/perelman.html).

[2] A. Besse, Einstein manifolds; Ergebnisse der Mathematik und ihrer Grenzgebiete 3. Folge Band 10; Springer-Verlag; ISBN 3-540-15279-2.

[3] J. Cheeger, G. Tian, On the cone structure at infinity of Ricci flat manifolds with Euclidean volume growth and quadratic curvature decay, Inv. Math. 118, (1994), 493-571.

[4] X. X. Chen, G. Tian, Ricci flow on Kähler-Einstein surfaces, Inv. Math. 147 (2002), 487-544.

[5] J. Eells, J. H. Sampson, Harmonic mappings of Riemannian manifolds, Ann. of Math. 86 (2) (1964) 109-160.

[6] R. Hamilton: Three-manifolds with positive Ricci curvature, J. Diff. Geom. 17 (1982) 225-306.

[7] R. Hamilton, Four-manifolds with positive curvature operator, J. Diff. Geom. 24 (1986), 153-179.

[8] R. Hamilton, A compactness property for solutions of the Ricci flow, Amer. J. Math. 117 (1995), 545-572. 
[9] R. Hamilton, The formation of singularities in the Ricci flow, Surveys in Differential Geometry, vol. 2, International Press, Cambridge, MA (1995) 7-136.

[10] R. Hamilton, Non-singular solutions of the Ricci flow on 3 manifolds, Communications in Analysis and Geometry, 7 (1999), 695-729.

[11] G. Perelman, The entropy formula for the Ricci flow and its geometric applications; arXiv:math.DG/0211159

[12] Rothaus, Logarithmic Sobolev inequality and spectrum, J. Dif. Anal. 42 (1981), 109-120.

[13] W. X. Shi, Ricci deformation of the metric on complete noncompact Riemannian manifolds, J. Diff. Geom. 30 (1989), 303-394.

[14] N. Sesum, G. Tian, X. Wang, Notes on Perelman's paper.

[15] L. Simon, Asymptotics for a class of non-linear evolution equations, with applications to geometric problems, Ann. Math. 118 (1983), 525571.

[16] M. Struwe, On the evolution of harmonic maps in higher dimensions, J. Diff. Geom. 28 (1988), 485-502.

[17] P. Topping, Diameter control under Ricci flow, http://www.maths.warwick.ac.uk/ topping/diamrf.pdf.

[18] Rugang Ye, Ricci flow, Einstein metrics and space forms, Transactions of the American Mathematical Society, 338 (2) (1993) 871-895. 